\title{
The Interface Region Imaging Spectrograph (IRIS)
}

B. De Pontieu • A.M. Title · J.R. Lemen - G.D. Kushner • D.J. Akin • B. Allard •

T. Berger $\cdot$ P. Boerner $\cdot$ M. Cheung $\cdot$ C. Chou $\cdot$ J.F. Drake $\cdot$ D.W. Duncan •

S. Freeland • G.F. Heyman • C. Hoffman • N.E. Hurlburt • R.W. Lindgren •

D. Mathur • R. Rehse • D. Sabolish • R. Seguin • C.J. Schrijver • T.D. Tarbell •

J.-P. Wülser • C.J. Wolfson • C. Yanari $\cdot$ J. Mudge $\cdot$ N. Nguyen-Phuc $\cdot$ R. Timmons •

R. van Bezooijen · I. Weingrod · R. Brookner • G. Butcher · B. Dougherty · J. Eder •

V. Knagenhjelm • S. Larsen • D. Mansir · L. Phan · P. Boyle · P.N. Cheimets •

E.E. DeLuca · L. Golub · R. Gates · E. Hertz • S. McKillop • S. Park · T. Perry •

W.A. Podgorski · K. Reeves · S. Saar • P. Testa • H. Tian • M. Weber • C. Dunn •

S. Eccles · S.A. Jaeggli · C.C. Kankelborg · K. Mashburn • N. Pust • L. Springer •

R. Carvalho · L. Kleint · J. Marmie · E. Mazmanian - T.M.D. Pereira • S. Sawyer •

J. Strong • S.P. Worden • M. Carlsson • V.H. Hansteen • J. Leenaarts • M. Wiesmann •

J. Aloise · K.-C. Chu • R.I. Bush • P.H. Scherrer • P. Brekke · J. Martinez-Sykora •

B.W. Lites - S.W. McIntosh • H. Uitenbroek · T.J. Okamoto • M.A. Gummin •

G. Auker · P. Jerram $\cdot$ P. Pool · N. Waltham

Received: 3 December 2013 / Accepted: 24 January 2014 / Published online: 13 February 2014

(C) The Author(s) 2014. This article is published with open access at Springerlink.com

\begin{abstract}
The Interface Region Imaging Spectrograph (IRIS) small explorer spacecraft provides simultaneous spectra and images of the photosphere, chromosphere, transition region, and corona with $0.33-0.4$ arcsec spatial resolution, two-second temporal resolution, and $1 \mathrm{~km} \mathrm{~s}^{-1}$ velocity resolution over a field-of-view of up to $175 \operatorname{arcsec} \times 175 \operatorname{arcsec}$.
\end{abstract}

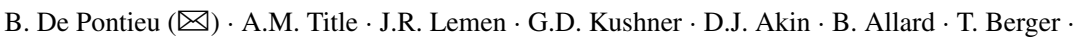

P. Boerner · M. Cheung · C. Chou · J.F. Drake · D.W. Duncan · S. Freeland · G.F. Heyman ·

C. Hoffman · N.E. Hurlburt · R.W. Lindgren · D. Mathur · R. Rehse · D. Sabolish · R. Seguin ·

C.J. Schrijver · T.D. Tarbell · J.-P. Wülser · C.J. Wolfson · C. Yanari · J. Martinez-Sykora

Lockheed Martin Solar \& Astrophysics Laboratory, Lockheed Martin Advanced Technology Center,

Org. A021S, Bldg. 252, 3251 Hanover St., Palo Alto, CA 94304, USA

e-mail: bdp@Imsal.com

B. De Pontieu · T.M.D. Pereira · M. Carlsson · V.H. Hansteen · J. Leenaarts · M. Wiesmann Institute of Theoretical Astrophysics, University of Oslo, P.O. Box 1029 Blindern, Oslo, Norway

T. Berger $\cdot$ H. Uitenbroek

National Solar Observatory, Sacramento Peak, P.O. Box 62, Sunspot, NM 88349-0062, USA

J. Mudge $\cdot$ N. Nguyen-Phuc $\cdot$ R. Timmons $\cdot$ R. van Bezooijen $\cdot$ I. Weingrod

Lockheed Martin Advanced Technology Center, Palo Alto, 3251 Hanover St., Palo Alto, CA 94304, USA

R. Brookner · G. Butcher · B. Dougherty · J. Eder · V. Knagenhjelm · S. Larsen · D. Mansir · L. Phan ·

P. Boyle

Lockheed Martin, 1111 Lockheed Martin Way, Sunnyvale, CA 94089, USA 
IRIS was launched into a Sun-synchronous orbit on 27 June 2013 using a Pegasus-XL rocket and consists of a 19-cm UV telescope that feeds a slit-based dual-bandpass imaging spectrograph. IRIS obtains spectra in passbands from $1332-1358 \AA$, $1389-1407 \AA$, and 2783-2834 $\AA$, including bright spectral lines formed in the chromosphere (Mg II h $2803 \AA$ and $\mathrm{Mg}$ II k $2796 \AA$ ) and transition region (C II 1334/1335 $\AA$ and Si IV $1394 / 1403 \AA$ ). Slit-jaw images in four different passbands (C II 1330, Si IV 1400, Mg II k 2796, and $\mathrm{Mg}$ II wing $2830 \AA$ A) can be taken simultaneously with spectral rasters that sample regions up to $130 \operatorname{arcsec} \times 175 \operatorname{arcsec}$ at a variety of spatial samplings (from 0.33 arcsec and up). IRIS is sensitive to emission from plasma at temperatures between $5000 \mathrm{~K}$ and $10 \mathrm{MK}$ and will advance our understanding of the flow of mass and energy through an interface region, formed by the chromosphere and transition region, between the photosphere and corona. This highly structured and dynamic region not only acts as the conduit of all mass and energy feeding into the corona and solar wind, it also requires an order of magnitude more energy to heat than the corona and solar wind combined. The IRIS investigation includes a strong numerical modeling component based on advanced radiative-MHD codes to facilitate interpretation of observations of this complex region. Approximately eight Gbytes of data (after compression) are acquired by

P.N. Cheimets · E.E. DeLuca $\cdot$ L. Golub · R. Gates $\cdot$ E. Hertz · S. McKillop · S. Park · T. Perry ·

W.A. Podgorski $\cdot$ K. Reeves $\cdot$ S. Saar $\cdot$ P. Testa $\cdot$ H. Tian $\cdot$ M. Weber

Harvard-Smithsonian Astrophysical Observatory, 60 Garden Street, Cambridge, MA 02138, USA

C. Dunn · S. Eccles · S.A. Jaeggli · C.C. Kankelborg · K. Mashburn · N. Pust · L. Springer

Department of Physics, Montana State University, Bozeman, P.O. Box 173840, Bozeman, MT 59717, USA

R. Carvalho · L. Kleint · J. Marmie · E. Mazmanian - T.M.D. Pereira · S. Sawyer · J. Strong ·

S.P. Worden

NASA Ames Research Center, Moffet Field, CA 94305, USA

L. Kleint · J. Martinez-Sykora

Bay Area Environmental Research Institute, 596 1st St West, Sonoma, CA 95476, USA

J. Aloise · K.-C. Chu · R.I. Bush · P.H. Scherrer

W.W. Hansen Experimental Physics Laboratory, Center for Space Science and Astrophysics, Stanford University, Stanford, CA 94305, USA

P. Brekke

Norwegian Space Centre, P.O. Box 113 Skøyen, 0212 Oslo, Norway

B.W. Lites · S.W. McIntosh

High Altitude Observatory, National Center for Atmospheric Research, P.O. Box 3000, Boulder, CO 80307, USA

T.J. Okamoto

ISAS/JAXA, Sagamihara, Kanagawa 252-5210, Japan

M.A. Gummin

Alias Aerospace, Inc., 1731 Saint Andrews Court, St. Helena, CA 94584, USA

G. Auker · P. Jerram · P. Pool

e2v technologies, 106 Waterhouse Lane, Chelmsford, Essex CM1 2QU, UK

N. Waltham

Rutherford Appleton Laboratory, Harwell Business Innovation Campus, Didcot, Oxon, OX11 0QX, UK 
IRIS each day and made available for unrestricted use within a few days of the observation.

Keywords Heating, chromospheric · Heating, coronal · Chromosphere, models · Chromosphere, active $\cdot$ Corona, active $\cdot$ Magnetic fields, chromosphere $\cdot$ Instrumentation and data management $\cdot$ Spectrum, ultraviolet

\section{Introduction}

The chromosphere and transition region (TR) form a complex interface region between the solar surface and corona. Almost all of the mechanical energy that drives solar activity and solar-atmospheric heating is converted into heat and radiation within this interface region, with only a small amount leaking through to power coronal heating and drive the solar wind. Understanding the chromosphere and TR is a foundational necessity for explaining the corona and heliosphere. They require a heating rate that is between one and two orders of magnitude larger than that of the corona. It is also here that we can find information on processes as diverse as the role of field-line braiding, interaction of the active-region and network field with the small-scale magnetic carpet, the interaction of emerging flux with the existing fields, wave propagation and mode conversion, mass supply to the corona and solar wind, and signatures of coronal processes through thermal conduction or energetic particles.

Despite the importance of the chromosphere and TR interface region for solar activity, the heating of the corona, and the genesis of the solar wind, this interface region remains poorly understood because it is highly complex and must be observed over a wide spectral range (from the visible to the EUV). As a result, it presents a challenging target for observers and modelers alike. The transition between high and low plasma $\beta$ occurs somewhere between the photosphere and corona, so that in the interface region, the magnetic field and plasma compete for dominance (with a variety of impacts on, e.g. waves, such as mode coupling, refraction, and reflection). Within this region, the density drops by six orders of magnitude, and the temperature rapidly increases from 5000 to 1 million $\mathrm{K}$, with strong gradients across the magnetic field evident from high-resolution images and spectra of the chromosphere (see, e.g., Figures 1, 2, 3, 4, 5, and 6).

The plasma transitions from partially ionized in the chromosphere to fully ionized in the corona and shows evidence of supersonic and super-Alfvénic motions. In addition, the chromosphere is partially opaque, with non-local thermodynamic equilibrium (non-LTE) effects dominating the radiative transfer, so that interpreting the radiation, and determining the local energy balance and ionization state, is non-intuitive and requires advanced computer models. The highly dynamic nature of the chromosphere, as observed with Hinode (Kosugi et al., 2007) and ground-based telescopes, further complicates attempts to better understand the interface region. This is both because high-cadence observations are required (better than $\approx 20$ seconds), and because the ionization states of some elements (e.g. hydrogen) react only slowly to changes in the energy balance, and thus depend on the history of the plasma.

The launch of the IRIS mission opens a new window into the complex physics of the interface region. The spectral ranges that IRIS observes have previously been studied at lower resolution using rockets (Bates et al., 1969; Fredga, 1969; Kohl and Parkinson, 1976; Allen and McAllister, 1978; Morrill and Korendyke, 2008; West et al., 2011; Dere, Bartoe, and Brueckner, 1984), balloons (Lemaire, 1969; Lemaire and Skumanich, 1973; Samain and Lemaire, 1985; Staath and Lemaire, 1995), or satellites (Doschek and Feldman, 1977; Bonnet et al., 1978; Woodgate et al., 1980; Roussel-Dupre and Shine, 1982; 

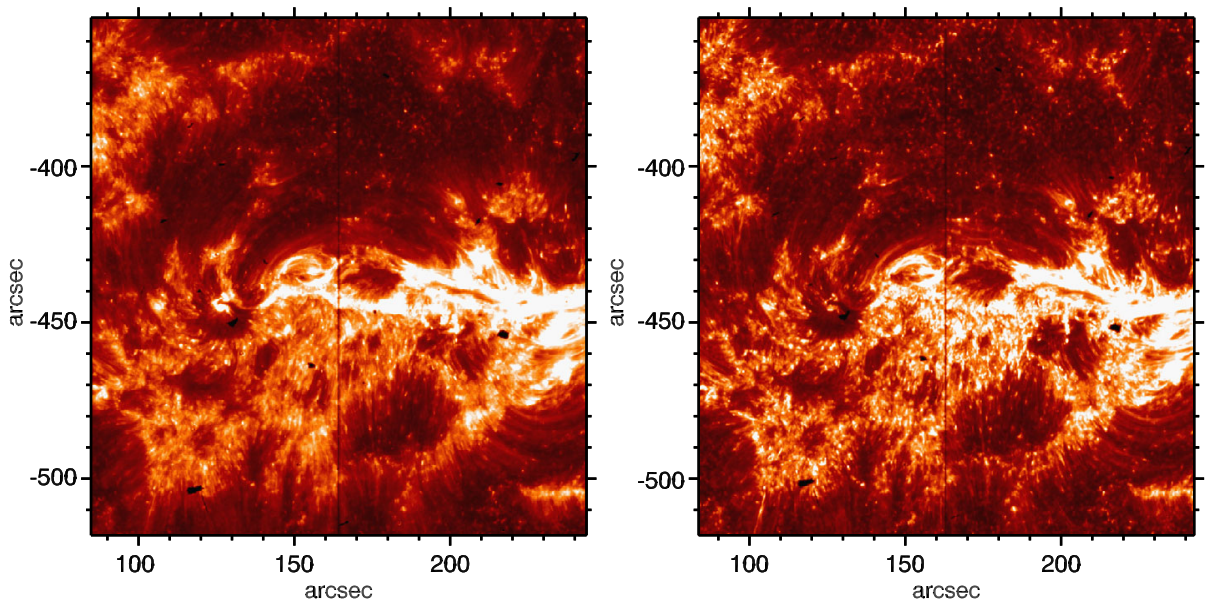

Figure 1 IRIS slit-jaw images (SJI) $1330 \AA$ and $1400 \AA$ of NOAA AR 11817 taken on 14 August 2013 at 18:50 UT. These images are sensitive to plasma of $10000-30000 \mathrm{~K}(1330 \AA)$ and $65000 \mathrm{~K}(1400 \AA)$ and show the upper chromosphere and low transition region. Both images also contain contributions from continuum that is formed in the low chromosphere. The dark vertical line in the middle of the images is the location of the slit. Corresponding spectra are shown in Figures 3, 4, and 5.
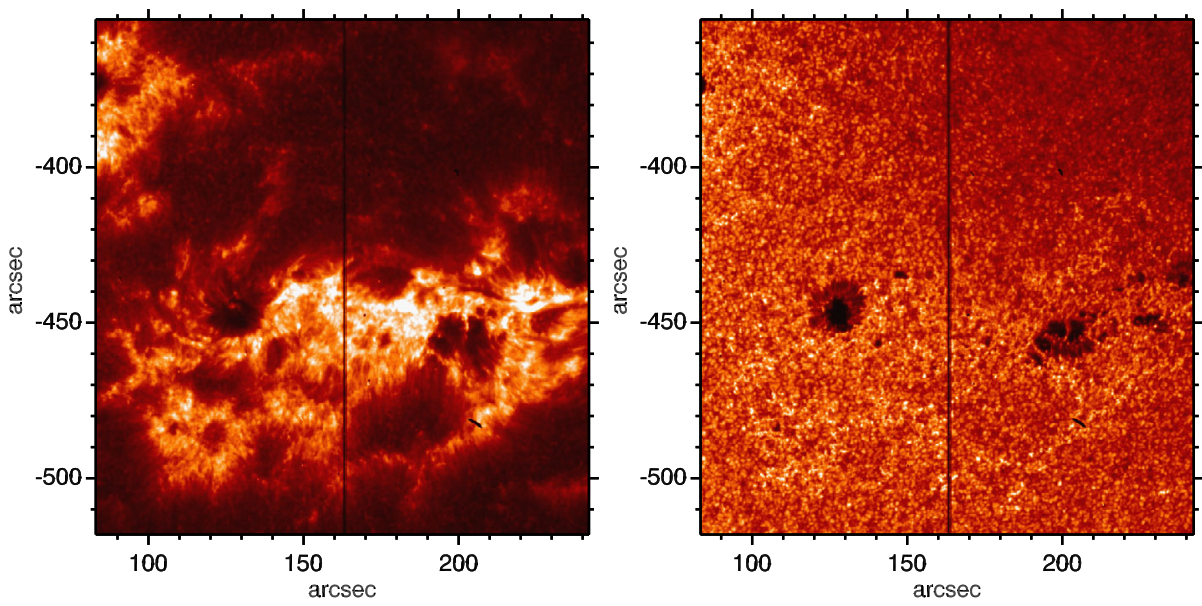

Figure 2 IRIS slit-jaw images (SJI) at $2796 \AA$ and $2830 \AA$ images of NOAA AR 11817 taken on 14 August 2013 at 18:50 UT. These images are sensitive to plasma of the upper chromosphere (2796 $\AA$ ) and upper photosphere $(2830 \AA)$. The upper-chromospheric image also contains contributions from the upper photosphere to the middle chromosphere, which are dominant in more quiet regions. The dark vertical line in the middle of the images is the location of the slit. Corresponding spectra are shown in Figures 3, 4, and 5.

Billings, Roussel-Dupre, and Francis, 1977; Poland and Tandberg-Hanssen, 1983; Kingston et al., 1982). IRIS draws on heritage solar instrumentation, such as the Transition Region and Coronal Explorer (TRACE: Handy et al., 1999), the Helioseismic and Magnetic Imager (HMI: Scherrer et al., 2012) and the Atmospheric Imaging Assembly (AIA: Lemen et al., 2012) onboard the Solar Dynamics Observatory (SDO: Pesnell, Thompson, and Chamberlin, 2012), and it exploits advances in novel, high-throughput, and high-resolution instru- 


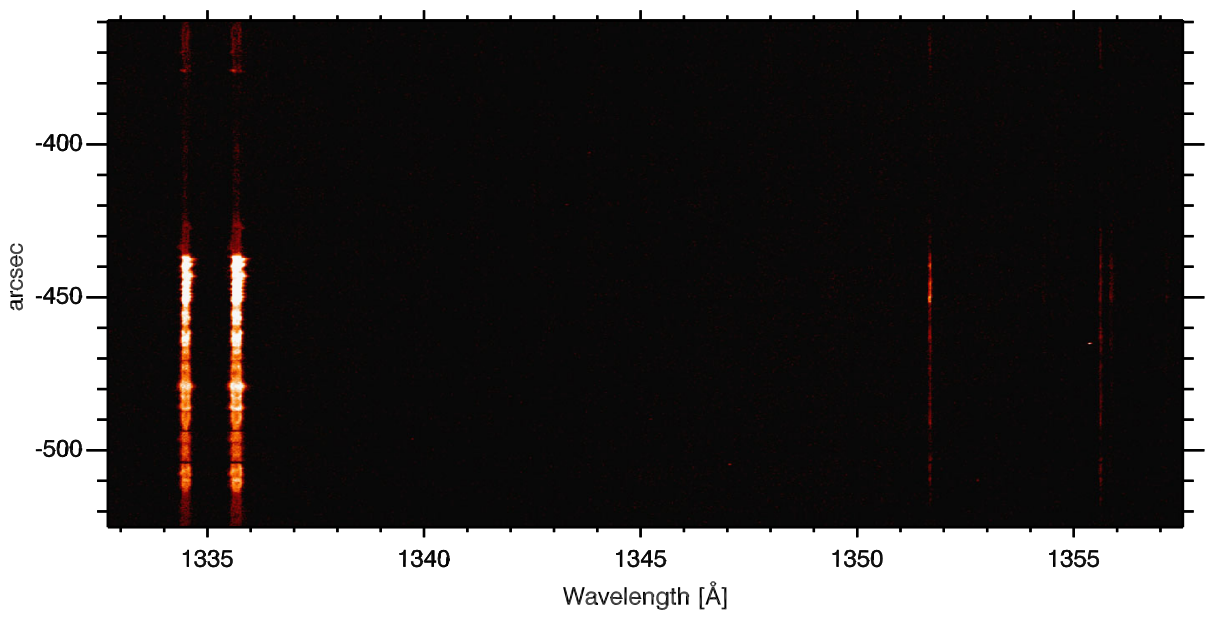

Figure 3 IRIS far ultra-violet (FUV) 1 spectrum of NOAA AR 11817 taken on 14 August 2013 at 18:50 UT. The two strong lines around 1334-1336 $\AA$ are C II lines that are formed in the upper chromosphere and low transition region. The lines longward of $1350 \AA$ are $\mathrm{Cl}$ I, O I, and C I lines that are formed in the low to middle chromosphere. Corresponding images are shown in Figures 1 and 2.

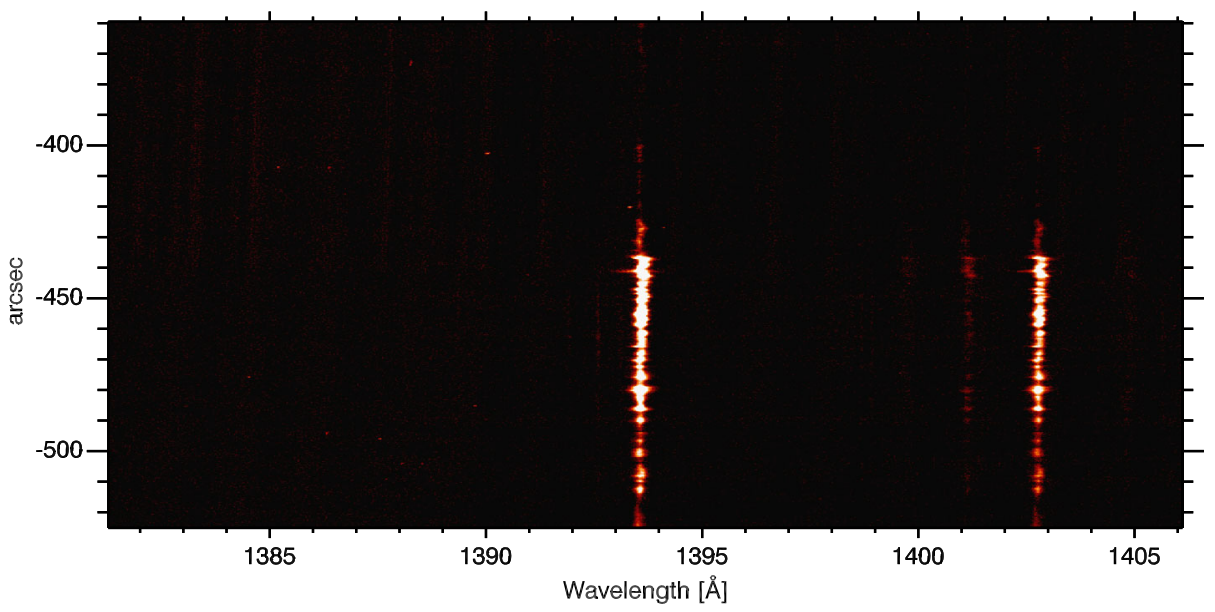

Figure 4 IRIS far ultra-violet (FUV) 2 spectrum of NOAA AR 11817 taken on 14 August 2013 at 18:50 UT. The strongest lines are Si IV lines, formed in the transition region $(65000 \mathrm{~K})$. The weak lines around the Si IV $1402 \AA$ line are O IV lines that are formed around $150000 \mathrm{~K}$ under equilibrium conditions. The very faint vertical lines in the upper half of the detector are an example of the noise pattern caused by electronic interference during camera readout (see Section 7.1). Corresponding images are shown in Figures 1 and 2.

mentation, efficient numerical simulation codes, and powerful, massively parallel supercomputers to aid interpretation of the data.

In Section 2 we give an overview of the IRIS observatory and its capabilities. We describe the science goals of the IRIS mission in Section 3 and the instrument in Section 4. The instrument observing approach and the day-to-day operations are described in Sections 5 and 6, respectively. We discuss the preliminary calibration of IRIS in Section 7, while the data pro- 


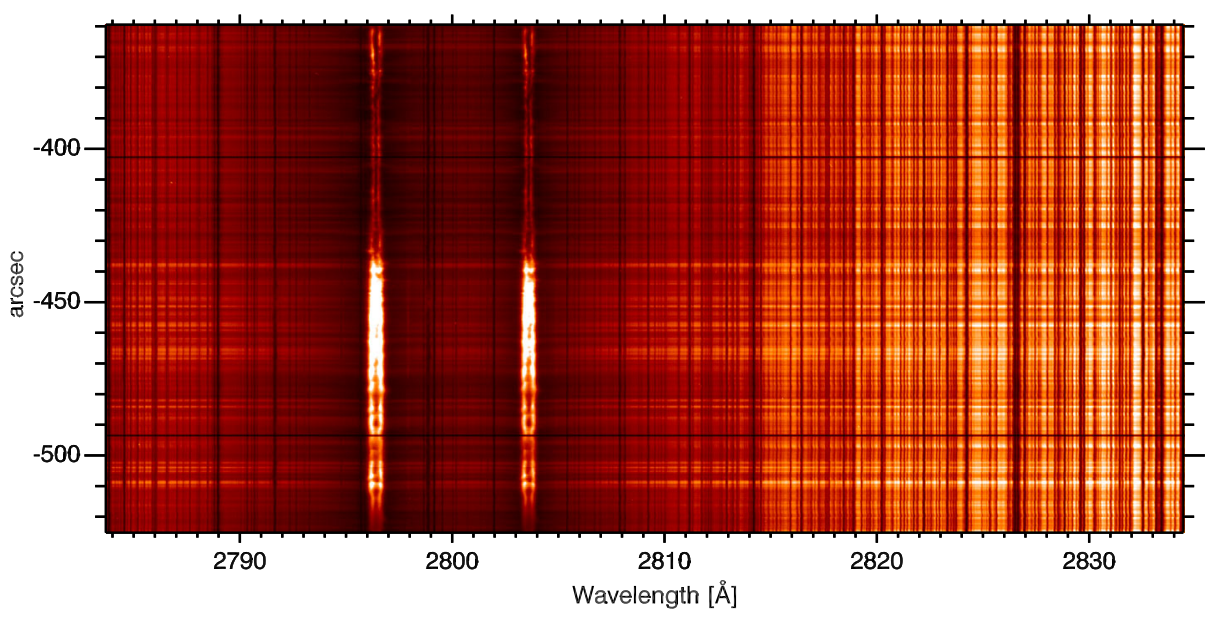

Figure 5 IRIS near ultra-violet spectrum of NOAA AR 11817 taken on 14 August 2013 at 18:50 UT. The two strong emission lines are Mg II k $2796 \AA ̊$ and Mg II h $2803 \AA$, both formed over a range of heights from the upper photosphere to the upper chromosphere. This wavelength range also contains a multitude of photospheric lines. The thin horizontal lines are fiducial marks that allow for easy co-alignment. Corresponding images are shown in Figures 1 and 2.
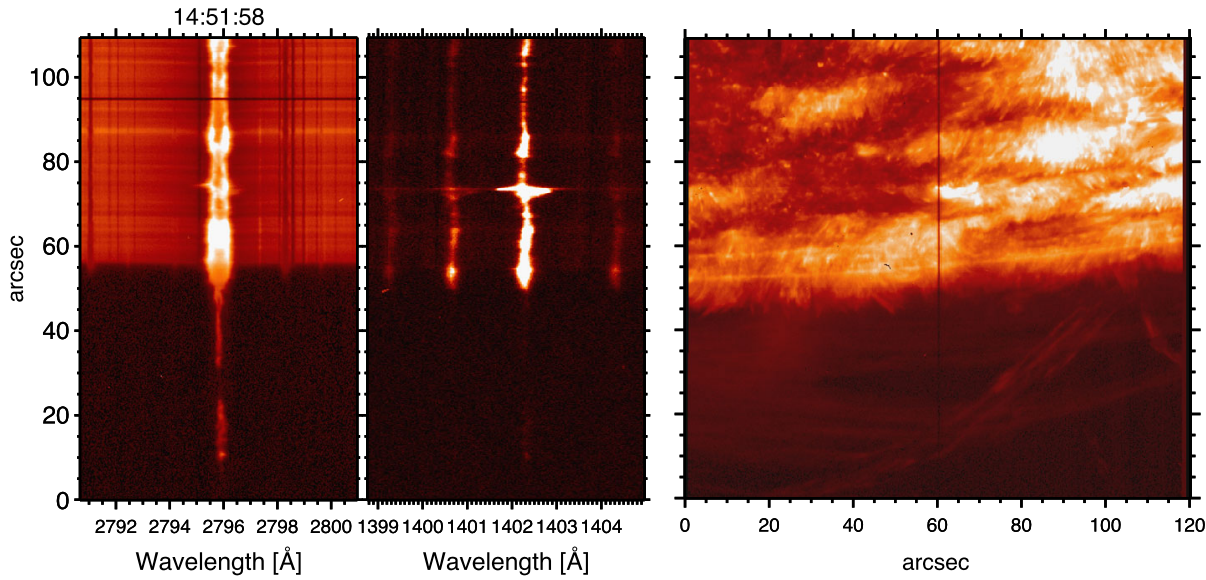

Figure 6 IRIS spectra of Mg II k $2796 \AA$ (left panel), Si IV 1402 A (middle panel), and 1400 SJI image (right panel) of an active region at the limb. The faint lines surrounding the bright Si IV line in the middle panel are O IV lines, which can be used to determine densities in the transition region. Note the strong blueand redward excursions in all spectral lines throughout the plage region. Off-limb the slit-jaw image captures spicules, coronal rain, and prominence material. The solar limb in the NUV spectrum (left panel) clearly shows the different range of formation heights for various spectral lines. The spacecraft was rolled by 90 degrees for these observations. The $x$ - and $y$-coordinates are shown to indicate the scale of the image and spectra only.

cessing is detailed in Section 8. We finish with a description of the numerical-simulations approach in the IRIS science investigation (Section 9). Our conclusions are given in Section 10 . 


\section{IRIS Observatory}

\subsection{IRIS Properties}

The conditions in the interface region present a significant challenge to observers. Observations with Hinode and ground-based instruments such as the Interferometric Bidimensional Spectrometer (IBIS: Cavallini, 2006) at the Dunn Solar Telescope (DST) and the Crisp Imaging Spectropolarimeter (CRISP: Scharmer et al., 2008) at the Swedish Solar Telescope (SST: Scharmer et al., 2003) indicate that to properly capture the dynamics, fine-scale structuring, and small-scale wave motions, observations at a cadence better than 20 seconds and a spatial resolution of better than 0.5 arcsec are required. To track the thermal evolution of the heating and cooling plasma in the interface region, observations need to simultaneously cover temperatures from the photosphere into the corona. To diagnose plasma conditions such as velocity, turbulence, non-thermal energy, or density, spectroscopic information needs to be obtained at high resolution and high signal-to-noise $(\mathrm{S} / \mathrm{N})$ ratios. Velocity fields must be determined to an accuracy of $1 \mathrm{~km} \mathrm{~s}^{-1}$ (e.g. through sub-pixel line centroiding), and to resolve non-thermal line broadening a spectral resolution on the order of $5-10 \mathrm{~km} \mathrm{~s}^{-1}$ is required. An instrument with these properties should be able to, e.g., quantify the properties of the waves that permeate this interface region (De Pontieu et al., 2007a, 2012; McIntosh et al., 2011; Okamoto and De Pontieu, 2011; McIntosh and De Pontieu, 2012; Sekse et al., 2013) and disentangle the complexities of the multiple spectral components that have been indirectly inferred using current spectroscopic data (De Pontieu et al., 2009; Bryans, Young, and Doschek, 2010; Tian et al., 2011; McIntosh et al., 2012).

The observational requirements given above drove the IRIS mission to provide the following essential capabilities:

- High spatial resolution (0.4 arcsec) spectroscopic and (context) imaging data over a field of view of at least 120 arcsec, providing diagnostics from the photosphere to the corona, with an emphasis on the chromosphere and transition region.

- A high signal-to-noise ratio for two-second exposures for a few select bright lines covering chromosphere and transition region, which allows a velocity determination with $1 \mathrm{~km} \mathrm{~s}^{-1}$ accuracy and $3 \mathrm{~km} \mathrm{~s}^{-1}$ spectral pixels.

- High-cadence spectral (20 second) and imaging (10 second) observations covering a small region of the Sun $(5 \times 120$ arcsec $)$ for periods of up to eight hours continuously.

- Eclipse-free observations for up to eight months per year with about $15 \mathrm{X}$-band passes per day and an average data rate of $0.7 \mathrm{Mbit} \mathrm{s}^{-1}$.

These capabilities are met by a design that includes the following:

- A 19-cm Cassegrain telescope that feeds a dual-range UV spectrograph (SG) and slit-jaw imager, with 0.16 arcsec pixels and four $2061 \times 1056$ CCDs.

- A slit-jaw imager that includes four passbands with two transition-region lines (C II $1335 \AA$ and Si IV $1400 \AA$ ), one chromospheric line (Mg II k $2796 \AA$ ), and one photospheric passband (2830 ̊), covering a field-of-view of $175 \operatorname{arcsec} \times 175 \operatorname{arcsec}$.

- A spectrograph with 0.33 arcsec wide and 175 arcsec long slit that covers FUV passbands from $1332 \AA$ to $1358 \AA$ and $1389 \AA$ to $1407 \AA$, and an NUV passband from $2783 \AA$ to $2835 \AA$. These passbands include lines formed over a wide range of temperatures from the photosphere $(5000 \mathrm{~K})$ to the corona (1 to 10 million $\mathrm{K}$ ).

- CCD detectors with a full well of 150000 electrons, with a camera-readout noise of $<20 \mathrm{e}^{-}$, and data compression that is nearly lossless. 
- Instrument-control software that allows for flexible rastering of the slit across the Sun (up to 21 arcmin from disk center), onboard summing, and various slit-jaw choices and cadences.

- A baseline cadence of three seconds per spectral-raster position, five seconds for slit-jaw images.

These capabilities enable IRIS to observe the thermal evolution of plasma from photospheric to coronal temperatures at the spatio-temporal resolution required for the highly dynamic interface region. The spectral, temporal, and spatial resolution and spectral coverage and effective areas of IRIS constitute significant advances over previous instrumentation. The IRIS throughput is more than an order of magnitude better than that of previous spectrographs such as the Solar Ultraviolet Measurements of Emitted Radiation instrument (SUMER: Wilhelm et al., 1995) or the Extreme ultraviolet Imaging Spectrograph (EIS: Culhane et al., 2007), both of which lack slit-jaw imaging for context. In its typical operational mode, IRIS obtains spectra about five to ten times faster than SUMER or EIS. The effective spatial resolution of IRIS is 0.4 arcsec, compared with the Nyquist-limited resolution of two arcsec of EIS or SUMER. IRIS enables imaging of the interface region with ten resolution elements for each of SDO/AIA's and 25 for each of SOHO/SUMER or Hinode/EIS. The velocity resolution of IRIS is more than three times better than SUMER and ten times better than EIS.

\subsection{Orbit}

IRIS was launched by an Orbital Space Systems Pegasus-XL rocket on 27 June 2013 into a Sun-synchronous, low-Earth orbit with an inclination of 97.9 degrees, perigee of $620 \mathrm{~km}$ and apogee of $670 \mathrm{~km}$ with a 0600 local time ascending node. For the first few years of operations, this orbit enables eclipse-free viewing from the beginning of February until the end of October. The rest of the year (November through January) the Earth blocks the IRIS view of the Sun for part of the orbit. The baseline plan is that instrument operations during eclipse season are not high priority, although power and thermal conditions will be assessed during the first eclipse season to determine the extent of IRIS operations during eclipse season.

IRIS passes through the South Atlantic Anomaly (SAA) and the northern and southern high-latitude zones (HLZs). While SAA passes lead to significantly increased numbers of energetic particle hits, the IRIS detectors have been shielded sufficiently so that flying through the HLZs has minimal impact on the quality of the data.

\subsection{Observatory}

The IRIS observatory mass is $183 \mathrm{~kg}$ with $87 \mathrm{~kg}$ for the instrument and $96 \mathrm{~kg}$ for the spacecraft bus. The IRIS spacecraft bus, designed and delivered by Lockheed Martin Civil Space (formerly LM Sensing and Exploration Systems), is a rigid design whose frame is machined from a single piece of aluminum with honeycomb aluminum forward and aft decks (Figure 7). Eight equipment bays house the spacecraft electronics boxes, reaction wheels, and the battery. The observatory measures about $2.18 \mathrm{~m}$ from the back of the spacecraft bus to the front of the telescope. The solar arrays deployed are $3.6 \mathrm{~m}$ tip to tip. The two solar arrays measure $0.6 \times 1.3 \mathrm{~m}$ each, with a total surface area of $1.7 \mathrm{~m}^{2}$ producing $340 \mathrm{~W}$. IRIS is three-axis stabilized. The attitude control system (ACS) is gyroless, using two star trackers, four reaction wheels, coarse and digital sun sensors, and a magnetometer. The instrument guide telescope provides a high-resolution pointing signal to the ACS during normal science 


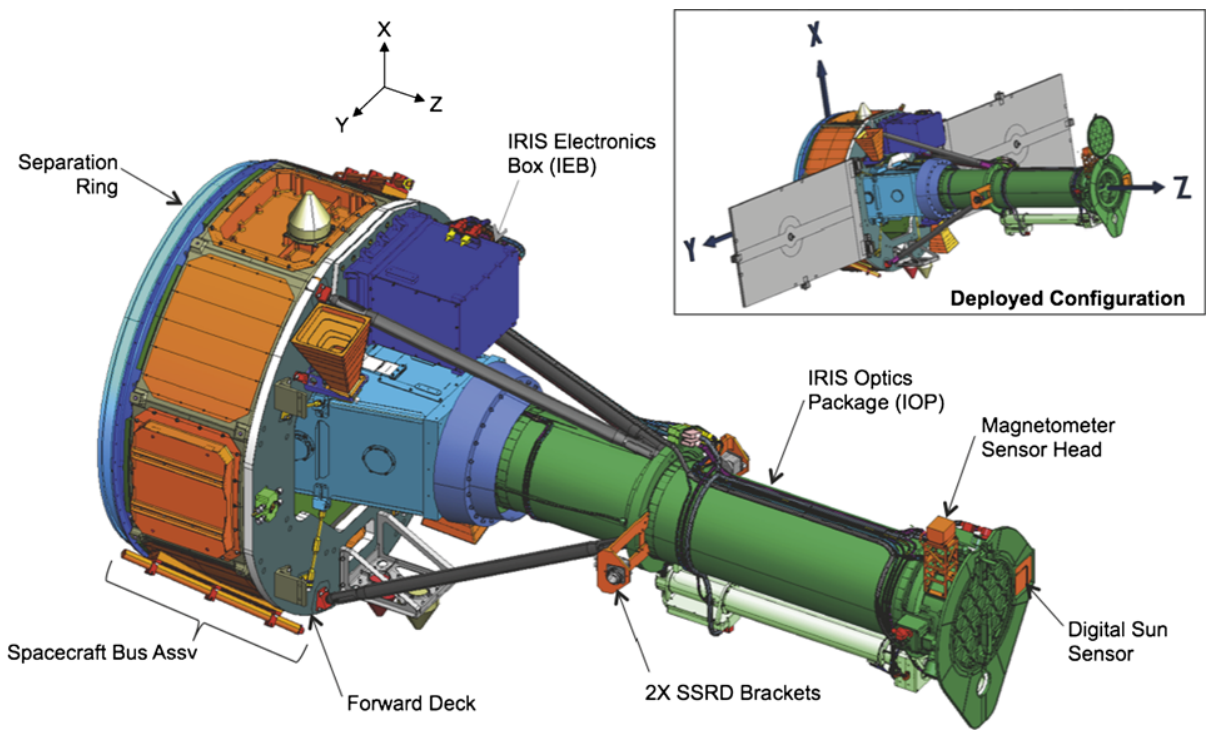

Figure 7 Schematic view of IRIS showing the 19-cm UV telescope, with and without solar panels (for clarity). Light from the Cassegrain telescope (green) is fed into the spectrograph box (light blue). The observatory measures $2.18 \mathrm{~m}$ from the back of the spacecraft bus to the front of the telescope.

operations. Magnetic-torque rods are used to manage the momentum of the reaction wheels, transferring energy to the Earth's magnetic field as needed. There is no propulsion system and there are no consumables onboard. The ACS can point the IRIS telescope boresight to any location on the solar disk or above the limb within 21 arcminutes of disk center, and roll the spacecraft (and thus, the spectrograph slit) up to $\pm 90^{\circ}$ (at $0^{\circ}$ the slit is oriented parallel to $\mathrm{N}-\mathrm{S}$ on the Sun). IRIS is equipped with two omnidirectional S-band antennas for uplinking of commands and downlinking of engineering data, and an X-band antenna for downlinking of science data. The S-band provides uplink at $2 \mathrm{kbits}^{-1}$ and downlink at $256 \mathrm{kbit} \mathrm{s}^{-1}$, whereas the X-band provides downlink at $15 \mathrm{Mbit} \mathrm{s}^{-1}$ including the overhead of Low Density Parity Checking (LDPC) 7/8 encoding. The effective downlink rate is $13 \mathrm{Mbit} \mathrm{s}^{-1}$ (excluding overhead) during up to 15 passes per day with the antennas of Kongsberg Satellite Services (KSAT) in Svalbard, Norway, as well as some passes from NASA's Near Earth Network (NEN) in Alaska and Wallops.

\section{Science Overview}

The IRIS investigation covers a broad range of scientific objectives that focus on three major questions that form the foundation of the IRIS investigation and have driven the requirements for the IRIS instrument design. Below we list an overview of the science issues covered by these three science questions.

\subsection{Which Types of Non-thermal Energy Dominate in the Chromosphere and Beyond?}

We still do not know which modes of non-thermal energy power the chromosphere, TR, corona, and solar wind. We know that waves, electrical currents, and magnetic reconnection 
all may release substantial energy, and that non-thermal particles, resistive dissipation, and wave damping occur. Yet, it remains unclear how much each of these contributes, how that depends on local conditions, and how and where the conversion of non-thermal to thermal energy happens in detail.

\subsubsection{Waves}

A variety of different wave modes have been observed in the past few years, from mostly acoustic modes and atmospheric gravity waves to Alfvén waves. However, there are still many unresolved questions regarding the role of these waves in the solar atmosphere.

For example, the role of acoustic power in the heating of the chromosphere and its potential role in the corona remains a puzzle, with some researchers reporting too little power and others arguing there should be enough. Most of the power in these modes lies in the range of the five-minute $p$-mode spectrum and the 10-15-minute time scale of granular evolution, but waves have been measured for periods as short as $\approx 25$ seconds, with some observations suggesting that these high-frequency waves carry a large, potentially dominant, energy flux. In addition, the normally evanescent $p$-mode oscillations may play a major role in the energy balance of the chromosphere in and around magnetic-field concentrations (Jefferies et al., 2006, so-called acoustic portals). The propagation of these sound waves along magneticfield lines leads to the formation of slow-mode magneto-acoustic shocks that propel plasma upwards to form so-called dynamic fibrils (Hansteen et al., 2006; De Pontieu et al., 2007b; Rouppe van der Voort et al., 2007; Kato et al., 2011). The role of these shocks in heating the magnetic chromosphere remains unknown but may be quite significant, based on analysis of spectra of the Ca II H line (Beck et al., 2008). Similarly, the enormous energy flux carried by atmospheric gravity waves in the internetwork (Straus et al., 2008) and the potential for mode coupling to other wave modes (e.g. Alfvén waves) around magnetic-field concentrations (Cally and Goossens, 2008) highlights another potential source of nonradiative energy.

Various types of mostly transverse MHD waves (such as Alfvén waves) are likely more efficient at traveling into the corona and solar wind, but their detailed energy budget remains mysterious. For example, relatively low-frequency $(\approx 1$ to $8 \mathrm{mHz})$ transverse and torsional (Alfvénic) motions have now been measured in the chromosphere and corona (Tomczyk et al., 2007; De Pontieu et al., 2007a, 2012; Tomczyk and McIntosh, 2009; McIntosh et al., 2011; Liu et al., 2011; Wedemeyer-Böhm et al., 2012; Su et al., 2013; Verwichte et al., 2013), but it is unclear what role higher-frequency waves play, how these waves are generated, and how they are dissipated.

IRIS spectroscopic measurements of waves as they propagate from the photosphere through the chromosphere and transition region into the corona will establish an energyflux budget, allowing us to disentangle wave mode coupling, dissipation, and propagation.

\subsubsection{Currents and Reconnection}

Establishing the energy associated with resistive dissipation of electrical currents within the chromosphere has been challenging. These currents are expected to be intense in compact fibrils. Any dissipation within the chromosphere can be expected to be associated with rapid temperature increases. Clearly, tracing the thermal evolution of chromospheric plasma holds large potential for providing information on how much electromagnetic energy is transformed into heat and how much energy propagates downwards from the corona as thermal energy or energetic particles in events ranging from microflares to X-class flares. Both of these types of processes are expected to occur, but we do not know their relative roles under a variety of conditions. 
The multi-fluid aspects introduced by the partial ionization of the chromosphere can dramatically alter the way in which electrical currents (and magnetic fields) are generated and dissipated (see, e.g., Arber, Haynes, and Leake, 2007) and thus provide a promising method of providing energy to the plasma. Various examples have recently been studied. For example, Goodman and Kazeminezhad (2010) focused on the dissipation of shortperiod shock waves from ion-neutral resistivity, whereas Fontenla, Peterson, and Harder (2008) studied the Farley-Bunemann instability (but see Gogoberidze et al., 2009), which depends critically on the ion magnetization (and thus the ion-neutral collision frequency and ionization degree). The importance of ion-neutral coupling has also been illustrated in advanced numerical models of the coupled solar atmosphere in which the chromosphere is self-consistently heated by ambipolar diffusion of currents generated through braiding of the magnetic field from magneto-convection (Martínez-Sykora, De Pontieu, and Hansteen, 2012).

A major unresolved issue is the role of reconnection of strong network or plage magnetic fields with the ubiquitous weak magnetic fields of mixed polarity on granular scales (Lites et al., 2008) in energizing the low solar atmosphere. Because the amount of weak flux that emerges on granular scales per day is much larger than the flux associated with active-region emergence over a full solar cycle, it is clear that if even a fraction of the weak fields reconnect with strong network or plage fields, the resulting energy release could be a dominant contributor to chromospheric or even coronal heating. Yet we do not know how much of this flux is dissipated in the solar atmosphere before most of it is recycled below the surface of the Sun.

IRIS measurements of chromospheric and coronal heating will help determine the sites of enhanced dissipation. Coordinated magnetic-field observations from ground- and spacebased telescopes will reveal the role of magnetic-field dissipation in the heating of the chromosphere and corona.

\subsection{How Does the Chromosphere Regulate the Mass and Energy Supply to the Corona and Heliosphere?}

The heating of the solar corona and the acceleration of the solar wind remain mysterious. IRIS contributes to our understanding by showing us in what form non-radiative energy is transmitted by the chromosphere into the corona. The studies described above help constrain wave-energy fluxes, while imaging of the chromosphere and photosphere combined with modeling constrains the forcing of the field into braids (see, e.g., Cirtain et al., 2013; Guerreiro, Hansteen, and De Pontieu, 2013) and twists by flows. IRIS also sheds light on how and where non-thermal energy is first released. Non-radiative energy deposited into the corona is lost both by radiation and transfer into the lower layers of the solar atmosphere. The transport to the lower layers can be thermal conduction, in which a relatively smooth temporal evolution can be expected, leading to evaporation of warming material from the top of the chromosphere. Alternatively, energy can propagate downward as energetic particles that can penetrate into the high chromosphere: such particle precipitations are likely more intermittent as they rapidly shift in position. Recent high-resolution observations in the transition region show direct evidence of this type of process (under some magnetic-field conditions) in active regions (Testa et al., 2013; Winebarger et al., 2013).

Other recent studies suggest that heating to coronal temperatures may occur primarily in the chromosphere and transition region, as evidenced, e.g. by chromospheric jets such as spicules (De Pontieu et al., 2011), and their faint coronal counterparts, visible only as slight enhancements in the blue wing of coronal spectral lines (De Pontieu et al., 2009). 
IRIS contributes to all of these issues by providing high-resolution and high signal-tonoise spectra and context images of spectral lines formed over a wide range of temperatures. Such measurements help reveal complex line shapes that arise along the line-of-sight in an interface region in which strong explosive upflows, gentle evaporative flows, and downflows all co-exist (McIntosh et al., 2012).

IRIS high-cadence observations significantly improve our ability to determine how much wave power is available to heat and accelerate the plasma in open-field regions at the base of the solar wind. Measurements of transverse and longitudinal waves, and their possible couplings to the solar wind, also provide constraints to models of the origin of turbulence in the heliosphere (Tu and Marsch, 1997; Axford et al., 1999; Cranmer, van Ballegooijen, and Edgar, 2007).

3.3. How Does Magnetic Flux and Matter Rise Through the Lower Atmosphere, and What Role Does Flux Emergence Play in Flares and Mass Ejections?

As magnetic field breaches the surface of the Sun, it begins to interact and reconnect with the existing field into which it intrudes. IRIS observations of flows help reveal the threedimensional flow patterns associated with flux emergence. The upflows of a few $\mathrm{km} \mathrm{s}^{-1}$ in the low chromosphere, the associated downdrafts of the draining arch-filament system at higher speeds, and the field expansion in the chromosphere and corona at Alfvén speeds are all measurable with IRIS. These observations will also help to uncover the reconnection processes that occur early in the life of active regions down to small granular-scale fields.

Emerging flux appears to play a key role in many flare phenomena. Observing flux as it breaches the surface and the lower chromosphere is an important diagnostic to understand flare initiation: chromospheric reconnection has been inferred in cases where emerging flux triggers a flare, and particle precipitation results in chromospheric ribbons both as tracers of coronal reconnections and as power sources for chromospheric evaporation. Observations of such flux emergence with Hinode suggest that the site of first energy release in a major flare occurred where strong electrical currents emerged. The thermal coverage and high resolution of IRIS allow insight into how the atmosphere evolves in the case of such a rapid energy release, and help constrain why flares are initiated where they are.

Spectroscopic observations of solar eruptions such as jets, flares, and CMEs can provide valuable information on the dynamics and plasma properties of the erupted materials (Harra et al., 2007; Tian et al., 2012; Young et al., 2013). IRIS's higher spectral resolution, higher cadence, and chromospheric and transition-region response make it a unique instrument for studying the initiation and early evolution of solar eruptions.

\section{Instrument Overview}

Portions of the IRIS instrument are described in some detail in pre-launch articles by Wülser et al. (2012) and Podgorski et al. (2012), and key parameters are provided in Table 1. Here we briefly summarize the instrument capabilities and refer the reader desiring more technical details to the above mentioned spectrograph and telescope articles.

The IRIS instrument uses a Cassegrain telescope with a 19-cm primary mirror and an active secondary mirror with a focus mechanism (Figures 7 and 8). The telescope has a field of view of about 3 arcmin $\times 3$ arcmin and feeds far-UV (FUV: from 1332 to $1407 \AA$ ) and near-UV (NUV: from 2783 to $2835 \AA$ ) light into a spectrograph box (Figures 3, 4, 5, and 6). Dielectric coatings throughout the optical path ensure that visible and IR radiation 
Table 1 IRIS instrument characteristics.

\begin{tabular}{|c|c|}
\hline Primary diameter & $19 \mathrm{~cm}$ \\
\hline Effective focal length & $6.895 \mathrm{~m}$ \\
\hline \multirow[t]{3}{*}{ Field of view } & $175 \times 175 \operatorname{arcsec}^{2}(\mathrm{SJI})$ \\
\hline & $0.33 \times 175 \operatorname{arcsec}^{2}(\mathrm{SG}-$ slit $)$ \\
\hline & $130 \times 175 \operatorname{arcsec}^{2}(\mathrm{SG}-$ raster $)$ \\
\hline Spatial scale (pixel) & $0.167 \operatorname{arcsec}$ \\
\hline \multirow[t]{2}{*}{ Spatial resolution } & $0.33 \operatorname{arcsec}(\mathrm{FUV})$ \\
\hline & $0.4 \operatorname{arcsec}(\mathrm{NUV})$ \\
\hline \multirow[t]{2}{*}{ Spectral scale (pixel) } & $12.8 \mathrm{mÅ}(\mathrm{FUV})$ \\
\hline & $25.6 \mathrm{mÅ}(\mathrm{NUV})$ \\
\hline \multirow[t]{2}{*}{ Spectral resolution } & 26 mÅ (FUV SG) \\
\hline & $53 \mathrm{mÅ}(\mathrm{NUV} \mathrm{SG})$ \\
\hline \multirow[t]{2}{*}{ Bandwidth } & $55 \AA ̊$ (FUV SJI) \\
\hline & $4 \AA ̊$ (NUV SJI) \\
\hline $\mathrm{CCD}$ detectors & $\begin{array}{l}\text { Four e2v } 2061 \times 1056 \text { pixels, thinned, } \\
\text { back-illuminated }\end{array}$ \\
\hline CCD cameras & $\begin{array}{l}\text { Two 4-port readout cameras (SDO flight } \\
\text { spares) }\end{array}$ \\
\hline Detector full well & 150000 electrons \\
\hline Typical exposure times & 0.5 to 30 seconds \\
\hline Flight Computer & BAe RAD 6000 \\
\hline \multicolumn{2}{|l|}{ Mass } \\
\hline Instrument & $87 \mathrm{~kg}$ \\
\hline Spacecraft & $96 \mathrm{~kg}$ \\
\hline Total & $183 \mathrm{~kg}$ \\
\hline \multicolumn{2}{|l|}{ Power } \\
\hline Instrument & $55 \mathrm{~W}$ \\
\hline Spacecraft & $247 \mathrm{~W}$ \\
\hline Total & $302 \mathrm{~W}$ \\
\hline \multicolumn{2}{|l|}{ Science Telemetry } \\
\hline Average downlink rate & 0.7 Mbit s $^{-1}$ \\
\hline $\mathrm{X}$-band downlink rate & 13 Mbit s $^{-1}$ \\
\hline Total data volume & $\approx 20$ Gbytes (uncompressed) per day \\
\hline
\end{tabular}

is suppressed. Most of the solar energy passes through the ULE substrate of the primary mirror and is radiated back into space. The FUV and NUV light follows several paths in the spectrograph box, as illustrated in Figure 9:

- Spectrograph (SG): light passes through a slit that is 0.33 arcsec wide and 175 arcsec long, and is dispersed onto either an NUV or an FUV grating. Light from the FUV grating is collected by two CCDs, light from the NUV grating is collected by a separate CCD (Table 2).

- Slit-jaw Imager (SJI): light is reflected off the reflective area around the slit (slit-jaw), passing through or reflecting off broadband filters in the filter wheel onto a fourth CCD to produce an image of the scene around the slit in six different filters (two for ground testing, four for solar images, Table 3). 
Table 2 IRIS spectrograph channels. Dispersion, Camera Electronics Box (CEB), and Effective Area (EA) vary for the three bandpasses.

\begin{tabular}{lllllllll}
\hline Band & $\begin{array}{l}\text { Wavelength } \\
{[\AA]}\end{array}$ & $\begin{array}{l}\text { Disp. } \\
{\left[\mathrm{m \AA} \mathrm{pix}^{-1}\right]}\end{array}$ & $\begin{array}{l}\text { FOV } \\
{\left[{ }^{\prime \prime}\right]}\end{array}$ & $\begin{array}{l}\text { Pixel } \\
{\left[{ }^{\prime \prime}\right]}\end{array}$ & CEB & Shutter & $\begin{array}{l}\text { EA } \\
{\left[\mathrm{cm}^{2}\right]}\end{array}$ & $\begin{array}{l}\text { Temp. } \\
{[\log T]}\end{array}$ \\
\hline FUV 1 & $1331.7-1358.4$ & 12.98 & 175 & 0.1663 & 1 & FUV SG & 1.6 & $3.7-7.0$ \\
FUV 2 & $1389.0-1407.0$ & 12.72 & 175 & 0.1663 & 1 & FUV SG & 2.2 & $3.7-5.2$ \\
NUV & $2782.7-2835.1$ & 25.46 & 175 & 0.1664 & 2 & NUV SG & 0.2 & $3.7-4.2$ \\
\hline
\end{tabular}

Table 3 IRIS slot channels. Filter-wheel positions can be either transmitting (T) or reflecting/mirrors (M).

\begin{tabular}{lllllllll}
\hline $\begin{array}{l}\text { Band- } \\
\text { pass }\end{array}$ & $\begin{array}{l}\text { Filter } \\
\text { wheel }\end{array}$ & Name & $\begin{array}{l}\text { Center } \\
{[\AA]}\end{array}$ & $\begin{array}{l}\text { Width } \\
{[\AA]}\end{array}$ & $\begin{array}{l}\text { FOV } \\
{\left[{ }^{\prime \prime} \times{ }^{\prime \prime}\right]}\end{array}$ & $\begin{array}{l}\text { Pix. } \\
{\left[{ }^{\prime \prime}\right]}\end{array}$ & $\begin{array}{l}\text { EA } \\
{\left[\mathrm{cm}^{2}\right]}\end{array}$ & $\begin{array}{l}\text { Temp. } \\
{[\log T]}\end{array}$ \\
\hline Glass & $1 \mathrm{~T}$ & 5000 & 5000 & broad & $175^{2}$ & 0.1679 & - & - \\
C II & $31 \mathrm{M}$ & 1330 & 1340 & 55 & $175^{2}$ & 0.1656 & 0.5 & $3.7-7.0$ \\
Mg II h/k & $61 \mathrm{~T}$ & 2796 & 2796 & 4 & $175^{2}$ & 0.1679 & 0.005 & $3.7-4.2$ \\
Si IV & $91 \mathrm{M}$ & 1400 & 1390 & 55 & $175^{2}$ & 0.1656 & 0.6 & $3.7-5.2$ \\
Mg II wing & $121 \mathrm{~T}$ & 2832 & 2830 & 4 & $175^{2}$ & 0.1679 & 0.004 & $3.7-3.8$ \\
Broad & $151 \mathrm{M}$ & $1600 \mathrm{~W}$ & 1370 & 90 & $175^{2}$ & 0.1656 & - & - \\
\hline
\end{tabular}

As indicated above, in the spectrograph focal plane there are four back-thinned CCD sensors with $2061 \times 1056$ pixels, three in the spectrograph path (two for FUV, one for NUV), and one CCD at the slit-jaw focal plane. Each 13- $\mu$ m pixel corresponds to 0.167 arcsec in the spatial direction, and $12.8 \mathrm{~m} \AA$ in the spectral direction for FUV spectra, and $25.5 \mathrm{~m} \AA$ for NUV spectra (the detailed plate scales for each channel are given in Tables 2 and 3). IRIS has an effective spatial resolution between 0.33 (FUV) and $0.4 \operatorname{arcsec}$ (NUV), and an effective spectral resolution of $26 \mathrm{~m} \AA$ in the FUV and $53 \mathrm{~m} \AA$ in the NUV. The four CCDs are controlled by two camera-electronics boxes (CEBs). Exposure times are controlled by three different mechanical shutters (FUV, NUV, and SJI). The IRIS telescopes also includes an active secondary mirror (driven by piezoelectric transducers or PZTs) for fine-scale pointing and image stabilization. The active secondary is pointed in response to signals from a guide telescope, which is mounted on the bottom of the telescope (Figure 7).

The field of view imaged by IRIS is $175 \times 175 \operatorname{arcsec}^{2}$ for the slit-jaw images. To produce a raster of spectra of the Sun, the IRIS active secondary mirror is scanned (using PZTs) in the direction perpendicular to the slit, causing different regions of the Sun to be exposed onto the slit. The slit scan range is \pm 65 arcsec, so that the maximum field of view of the IRIS rasters is $130 \times 175 \operatorname{arcsec}^{2}$ for the SG. The IRIS slit is nominally oriented parallel to the solar rotation axis (solar North), but the spacecraft can be rolled to any angle between $-90^{\circ}$ and $+90^{\circ}$ from solar North for extended periods of time.

Many parts of the IRIS instrument are based on heritage from TRACE, AIA, and HMI, as noted below. Extensive use of designs and parts from these previous missions reduced the need for lengthy life testing (for mechanisms) and enabled the modest schedule and budget of a small explorer to be achieved (45 months from start of Phase B until launch).

\subsection{Telescope}

The IRIS telescope is a 19-cm Cassegrain telescope with an active secondary mirror. It is based on the AIA telescope design, but has a longer focal length and uses a differ- 


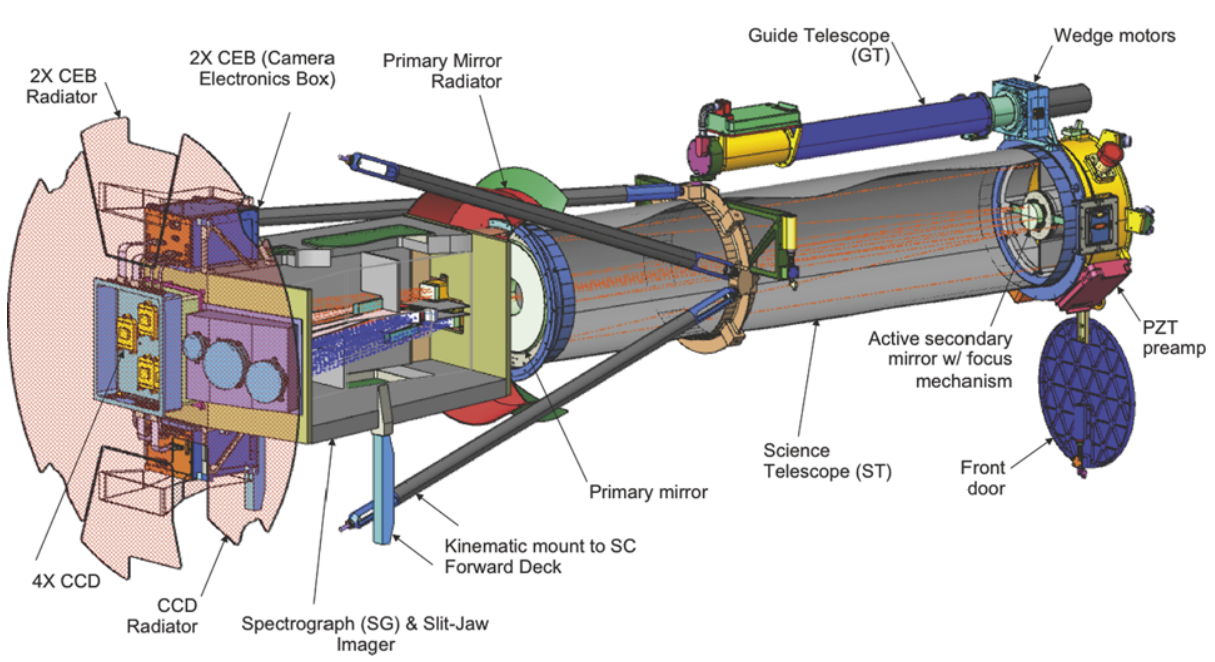

Figure 8 Conceptual design of the IRIS instrument. Sunlight enters from the right. For the flight design, the telescope and guide-telescope assemblies are rotated 180 degrees about the instrument axis relative to the spectrograph box (see Figure 7). The telescope measures $2.18 \mathrm{~m}$ from the CEB radiator to the front of the telescope.

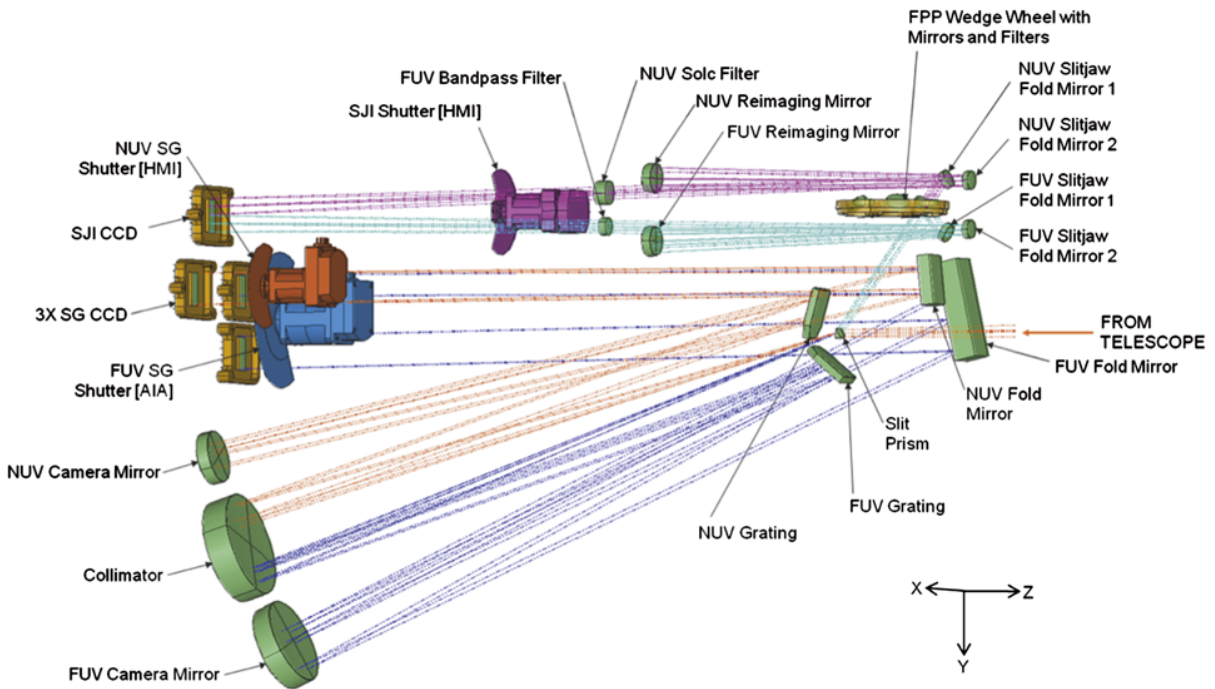

Figure 9 Path taken by light in the FUV spectrograph (dark blue), NUV spectrograph (orange), FUV slit-jaw (light blue), and NUV slit-jaw (purple) path. The distance between the FUV fold mirror and the SG CCDs is $0.63 \mathrm{~m}$.

ent thermal approach (Park et al., 2012; Hertz et al., 2012). Unlike the AIA design, sunlight enters the IRIS telescope unattenuated. At the primary mirror, most of the visible and infrared light passes through the transparent ULE mirror substrate and is collected by a heat sink. The front of the primary mirror has a dielectric coating that reflects UV light to the secondary mirror, which is subsequently focused on the spectrograph slit. The telescope is described in more detail by Podgorski et al. (2012). 
Table 4 Thermal coverage of IRIS spectrograph.

\begin{tabular}{|c|c|c|c|c|c|}
\hline Ion & $\begin{array}{l}\text { Wavelength } \\
{[\AA]}\end{array}$ & 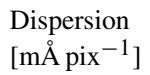 & $\begin{array}{l}\log T \\
{[\log \mathrm{K}]}\end{array}$ & Passband & CEB \\
\hline Mg II wing & 2820 & 25.46 & $3.7-3.9$ & NUV & 2 \\
\hline O I & 1355.6 & 12.98 & 3.8 & FUV 1 & 1 \\
\hline $\mathrm{Mg}$ II h & 2803.5 & 25.46 & 4.0 & NUV & 2 \\
\hline Mg II k & 2796.4 & 25.46 & 4.0 & NUV & 2 \\
\hline C II & 1334.5 & 12.98 & 4.3 & FUV 1 & 1 \\
\hline C II & 1335.7 & 12.98 & 4.3 & FUV 1 & 1 \\
\hline Si IV & 1402.8 & 12.72 & 4.8 & FUV 2 & 1 \\
\hline Si IV & 1393.8 & 12.72 & 4.8 & FUV 2 & 1 \\
\hline O IV & 1399.8 & 12.72 & 5.2 & FUV 2 & 1 \\
\hline O IV & 1401.2 & 12.72 & 5.2 & FUV 2 & 1 \\
\hline Fe XII & 1349.4 & 12.98 & 6.2 & FUV 1 & 1 \\
\hline Fe XXI & 1354.1 & 12.98 & 7.0 & FUV 1 & 1 \\
\hline
\end{tabular}

\subsection{Spectrograph}

The telescope feeds light into the spectrograph box (Figure 8), which contains the CzernyTurner spectrograph (Figure 9). The light from the telescope is focused on the slit assembly. The slit assembly is a prism that has a reflective coating, which also contains the slit. The reflective coating directs the light into the slit-jaw imager path. Light that goes through the slit into the prism is dispersed, directing FUV light in the $1332-1407 \AA$ range and NUV light in the 2783-2835 $\AA$ wavelength range onto separate parts of the collimator mirror. The slit/pre-disperser prism assembly ensures that both FUV and NUV passbands image the same region on the Sun within the $1 / 3 \times 175 \operatorname{arcsec}^{2}$ entrance slit.

After the collimator, the FUV and NUV SG spectrograph beams are fed to separate gratings, camera mirrors, and detectors (Table 2 and Figure 9). The FUV and NUV gratings, fabricated by Horiba Jobin-Yvon, have a groove density of 3600 lines $\mathrm{mm}^{-1}$ and are described in more detail by Wülser et al. (2012). The FUV and NUV SG beams have separate shutters and are recorded onto three separate CCDs: two for the FUV and one for the NUV. The two FUV CCDs observe two separate wavelength ranges: one that includes two bright C II lines (1332-1358 $)$ ), and another that contains Si IV and O IV lines (1389-1407 $⿱$ ). These two FUV CCDs are controlled by the same CEB and read out as if they were one CCD. The NUV passband from $2783-2835 \AA$ is recorded by a CCD that is controlled by a different CEB (which also reads out the SJI CCD).

IRIS spatial rasters are formed by scanning across the solar surface using the PZTs to change the orientation of the secondary mirror. Typical observing programs include both the FUV and NUV SG passband. The FUV and NUV spectral bandpasses cover spectral lines and continua that in the solar atmosphere are formed over a range of temperatures $\log T[\mathrm{~K}]=3.7-7$. Table 4 describes these lines in more detail. The brightest lines in the SG are the C II lines around $1335 \AA$ A, Si IV $1394 \AA$, Si IV $1403 \AA$, Mg II k $2796 \AA$, and Mg II h $2803 \AA$. These are the lines that are included in routine, high-cadence raster scans where exposure times are of the order of two seconds. The O IV, Fe XII, and Fe XXI lines are fainter and require longer exposure times. 


\subsection{Slit-Jaw Imager}

The slit-jaw images that are reflected off the slit/prism assembly next reach the filter wheel. The filter wheel includes six different filters (four for solar applications, and two for ground testing: Table 3). The filter wheel can be rotated to place any one of the filters in the beam. The NUV filters are all transmitting, whereas the FUV filters are reflective, ensuring a different path for the NUV and FUV SJI beams. Each of these beams includes separate re-imaging and fold mirrors. Both beams encounter the same shutter mechanism and are recorded on the same CCD, with one half observing the NUV SJI images, and the other half the FUV SJI images.

The FUV beam includes a fixed FUV bandpass filter to block light with longer wavelengths. The NUV beam includes a Šolc filter with a free spectral range of $33 \AA$ to reduce the near-UV bandwidth to $3.6 \AA$ (Berger et al., 2012). The Mg II k and Mg II wing SJI filter options are realized by combining a broader interference filter $(\approx 15 \AA)$ in the filter wheel with the narrow-band Šolc filter.

The four solar SJI filter options are dominated by emission from C II 1334/1335 A, Si IV 1394/1403 $\AA$, Mg II k $2796 \AA$, and the wing of Mg II around $2830 \AA$, respectively. The relatively broad passbands imply that contributions from continuum or wing emission are significant and, depending on solar conditions, can be dominant. Nevertheless, the bright lines are expected to contribute significantly to the SJI images. The SJI images were chosen to provide diagnostics over a wide temperature range, as described in Table 3. The C II SJI filter images may include emission from the Fe XXI line under flaring conditions.

To enable solid co-alignment between the various SJI and SG channels, fiducial marks have been added to the slit. These are gaps two pixels long along the slit, one in the top half of the CCD and one in the bottom half of the CCD. These fiducial marks show up as dark features in the spectra and as bright regions in the slit portion of the SJI images.

\subsection{CCD Detector and Camera System}

The IRIS CCDs are custom-designed e2v devices (CCD267) that are similar to the AIA CCDs and are electrically compatible with the SDO camera design. The CCDs are backthinned and back-illuminated with a pixel size of $13 \mu \mathrm{m}$. Each CCD has $2061 \times 1056$ pixels and two readout amplifiers that enable both halves of the CCD to be read out simultaneously. The IRIS CCDs have been treated with a e $2 \mathrm{v}$ proprietary backside-enhancement process, which enables a quantum efficiency of about $31 \%$ at $1400 \AA$. The enhancement treatment results in an annealing pattern, visible in CCD flat-field images as an approximately rectangular grid of lines, which show sensitivity differences on the order of 5-10\% (Wülser et al., 2012).

The IRIS cameras are flight spares from SDO (AIA and HMI). They were developed at Rutherford Appleton Laboratory and are described in more detail by Lemen et al. (2012). Each camera has four read ports and, for SDO, each CEB was dedicated to a four-port AIA or HMI CCD. For IRIS, one CEB is used to read two CCD267 devices, with two ports per device. CEB 1 reads out simultaneously the four amplifiers from the FUV 1 and FUV 2 spectrograph CCDs. CEB 2 reads the two ports of the NUV SG CCD and the two ports of the slit-jaw imager CCD. The data are read out at two Mpixels s ${ }^{-1}$ through each interface, with less than 20 electrons of read noise. The cameras communicate with the IRIS electronics box through an IEEE 1355/SpaceWire link. The camera has programmable control of the waveform generator to provide different operating modes: full frame or subfield readout, gain and pedestal offset, flush, on-board summing, and the so-called inhibit skip mode (which allows very rapid repeats of exposures by skipping the full readout, but thus leaving some charge on the CCD). 


\subsection{Guide Telescope and Image Stabilization System}

The IRIS instrument has a guide telescope (GT) and image-stabilization system (ISS) that are based on the systems used for TRACE and AIA. In coordination with the spacecraft Attitude Control System (ACS), the GT allows pointing to chosen solar targets. The ACS can also roll the spacecraft to any angle in the range $\pm 90^{\circ}$ and provides short-term pointing stability to arcsec or better accuracy, using the error signals from the GT. The ISS removes faster jitter up to about $25 \mathrm{~Hz}$ and provides pointing stability down to 0.05 arcsec root-meansquared (RMS).

The GT consists of an achromatic refractor with an entrance filter that has a bandpass centered at $5700 \AA$ with a FWHM of $500 \AA$. Four redundant photo-diodes positioned behind an occulter measure the position of the solar limb. Intensity differences between opposite diodes are used to derive a displacement error signal with a linear range greater than \pm 80 arcsec. These analog error signals in the pitch and yaw directions are sent to the three piezoelectric transducers that tilt the secondary mirror of the main telescope. The IRIS flight software samples the GT error signals at a $32 \mathrm{~Hz}$ rate with a 12-bit ADC for 0.06 arcsec resolution. These are averaged and sent to the ACS at $5 \mathrm{~Hz}$, which controls the spacecraft pointing closed-loop to null these errors in pitch and yaw when in Fine Sun Pointing mode. The ACS uses dual star trackers to control roll and also for coarser pointing in pitch and yaw, when the GT is not in use (also known as Inertial Sun Pointing mode).

A pair of independently rotatable optical wedges (Risley prisms) are mounted between the entrance filter and the objective lens so that the GT can be oriented off the optical axis of the main IRIS telescope by up to 21 arcmin. Since the ACS continually adjusts the pointing to keep the GT solar image centered on the four diodes, this means that the main IRIS telescope is off-pointed from disk center to a chosen offset location. The hollow-core wedge motors are based on HMI designs and rotate the wedge prisms in discrete steps, so that the possible pointing locations are a grid in polar coordinates with a maximum spacing of 16 arcsec. Moving from one position on the solar disk to another can be done in Fine Sun Pointing mode, with the wedges moving in steps and the ACS following the desired pointing. This typically takes about two minutes and is less than six minutes in the longest cases. Fine-scale pointing below the wedge-grid resolution is done using the PZTs of the secondary mirror for any pointing offset that cannot be reached because of the discretization of the wedge-motor positions.

The error signals sent to the PZTs tilt the mirror and keep the solar image stably positioned on the slit to a precision of $<0.05$ arcsec. Motions of the three PZTs are constrained so that the mirror only tilts, and telescope focus is maintained. The ISS is an open-loop control system in the sense that the error signal is derived from the GT rather than from motions of the image in the telescope focal plane. Therefore, careful calibration is needed to match the voltage scale of the PZT actuators with that of the GT error signals. In addition, the PZT actuators are part of a closed-loop servo in which the feedback signals are derived from strain gauges incorporated into the actuators. Using the strain gauges, accurate and repeatable image offsets can be commanded while jitter compensation continues. The PZTs have a linear range of \pm 80 arcsec and a response time of $25 \mathrm{~ms}$. We reserve $\pm 65 \operatorname{arcsec}$ for raster scans and the rest for a combination of jitter correction, transients caused by momentum management or filter-wheel motion, orbital wobble, and wedge-motor granularity.

The performance of the ACS and ISS has been measured on-orbit, showing that the shortterm pointing stability is usually better than 0.25 arcsec RMS before correction by the ISS, and better than 0.05 arcsec after correction. The worst transients are caused by large filterwheel moves and momentum management of the reaction wheels. A filter-wheel move of 
$180^{\circ}$ causes a rigid rotation of the spacecraft of three arcsec over 0.5 seconds, followed by an ACS response with an overshoot of similar magnitude lasting 10-15 seconds. The transient is only in the direction perpendicular to the slit $\left(\mathrm{E}-\mathrm{W}\right.$ when the spacecraft is at $0^{\circ}$ roll angle, i.e. aligned $\mathrm{N}-\mathrm{S}$ ) and is attenuated by the ISS to $<0.10$ arcsec. Momentum management transients occur when the magnetic torquers are used to reduce reaction-wheel speeds. This occurs roughly once per orbit, lasts one to two minutes, and causes pointing transients of a few arcsec at most, which are slow enough that the ISS reduces the disturbance to a level similar to that of the filter-wheel transient. Since the GT does not sense rotation of the solar disk about its center, the GT does not measure spacecraft-roll errors. When the science telescope is pointing away from disk center, roll errors translate into line-of-sight pointing errors in the azimuthal direction, with a magnitude of $17 \operatorname{arcsec}$ for $1^{\circ}$ of roll error. The ACS roll errors are usually less than 20 arcsec, so these are negligible. Occasionally, a short outage occurs during the handover from one star tracker to the other, and this causes a roll error that results in a pointing transient of about one arcsec if observing at the limb.

The ACS/ISS system allows us to point anywhere on the Sun (within 21 arcminutes of disk center) with an accuracy of about five arcsec (caused by uncertainty in the wedge motor positions).

As expected from pre-launch analyses, thermal bending of the GT mounting causes a slowly varying pointing wobble that is in phase with the orbital thermal conditions (i.e. the satellite orientation with respect to the Earth's albedo). This wobble is on the order of a few arcsec over the course of one orbit and is relatively stable from day-to-day. The pointing wobble is nulled out by using an orbital wobble table (see below) that includes an additional correction signal for the PZTs that is applied as a function of time since the last ascending node. On-orbit tests show that the correction signal typically reduces the residual wobble to less than 0.3 arcsec.

\subsection{Mechanisms}

IRIS contains a total of eight mechanisms consisting of five distinct types. The IRIS telescope has a front door, focus, filter wheel, three shutter mechanisms, and the two GT wedge motors (discussed earlier).

The front door is designed to keep the interior of the telescope sealed from any contaminants during launch. It is based on the design for AIA in which the door is latched shut with a high-output paraffin actuator. The door hinge is spring-loaded and the door is slightly bowed to promote the 180-degree opening when on orbit. The IRIS door was successfully opened at the end of the bake-out period, about three weeks after launch.

The IRIS telescope has a focus mechanism that is based on the AIA focus mechanism, and it adjusts the position of the secondary mirror relative to the primary along the optical axis by up to $1277 \mu \mathrm{m}$ in $2.28 \mu \mathrm{m}$ steps. Given the magnification factor of five, this corresponds to approximately $57 \mu \mathrm{m}$ change in focus per step. The focus can be adjusted as often as every set of exposures, but this is not necessary for routine science observations, and is only used for some calibrations.

The filter-wheel mechanism is based on the HMI focus mechanism. It consists of a thin, brushless, DC motor manufactured by $\mathrm{H}$. Magnetics to which is added an optical encoder. The mechanism is operated as a stepper motor with 180 steps per revolution. The motor has six primary positions, and a move between positions (i.e. 30 steps per position, Table 3 ) requires approximately 0.18 seconds per position, plus an additional 0.07 seconds of overhead.

The shutters are based on the designs for the AIA and HMI shutters. Three shutters are installed: one each for FUV, NUV, and SJI. The main difference between these shutters is 
Table 5 IRIS sequencer tables. The sequencer tables have a hierarchical structure. OBS tables are the "highest" level and they "call" FRM tables, which in turn "call” FDB and CRS tables.

\begin{tabular}{lll}
\hline Table name & Acronym & Definition \\
\hline Camera Readout Structure & CRS & $\begin{array}{l}\text { CCD regions (spectral lines and field of view), } \\
\text { Summing modes } \\
\text { CRS, Exposure time, Compression parameters, } \\
\text { Lrame Definition Block }\end{array}$ \\
Frame List & FRM & $\begin{array}{l}\text { Time, FDB, Repeat Count, PZT offset (raster scan), } \\
\text { Focus, Slit-jaw bandpass, Cadence }\end{array}$ \\
Observing List & OBS & Time, FRM, Repeat Count, Cadence, PZT offset \\
\hline
\end{tabular}

that the FUV shutter is significantly larger than the other two shutters so that it can cover the two FUV CCDs. The minimum exposure times that are supported are $112 \mathrm{~ms}$ for the FUV and $36 \mathrm{~ms}$ for both the SJI and NUV.

\subsection{Electronics}

The IRIS electronics are based on heritage from the AIA electronics box. The IRIS electronics box (IEB) provides power and housekeeping interfaces to the spacecraft in addition to the science-data interface by way of two redundant IEEE 1355 SpaceWire ports. A BAe RAD6000 CPU supports the flight software, which is responsible for receiving commands from the spacecraft and controls the flow of housekeeping data and science data to the spacecraft. It is also responsible for the interfaces to the mechanisms, the heaters, the guide telescope, and the cameras. The portion of the flight software that controls the science observing program is called the instrument sequencer, and is described in the next section.

\section{Instrument Sequencer}

\subsection{Sequencer Overview}

IRIS observations are controlled by the instrument-sequencer portion of the flight software, which makes use of a series of tables that are prepared on the ground, checked into a database, and uploaded to the spacecraft as needed. Through these tables, the observational goals are achieved by specifying the number, order, and cadence of images and spectra to be acquired. The tables specify how the flight software controls the mechanisms, cameras, and the data-processing chain that delivers the data to the spacecraft. There are four main types of sequencer tables (see Table 5), which have a hierarchical structure. The lowest level are the Camera Readout Structure (CRS) and Frame Definition Block (FDB) tables, which control details for reading out the CCD. The Frame List (FRM) controls the timing and order in which CCD frames are acquired. The highest level is the Observing List or OBS table, which contains a science program made up of various FRMs. Observing lists are scheduled in a timeline that is typically uploaded on a daily basis.

The CRS, FDB, FRM, and OBS tables contain all of the information required to acquire an image or spectrum:

i) CRS table data controls which data are read out from the CCD and sent on to the data compression/high rate interface (DC/HRI) board. 


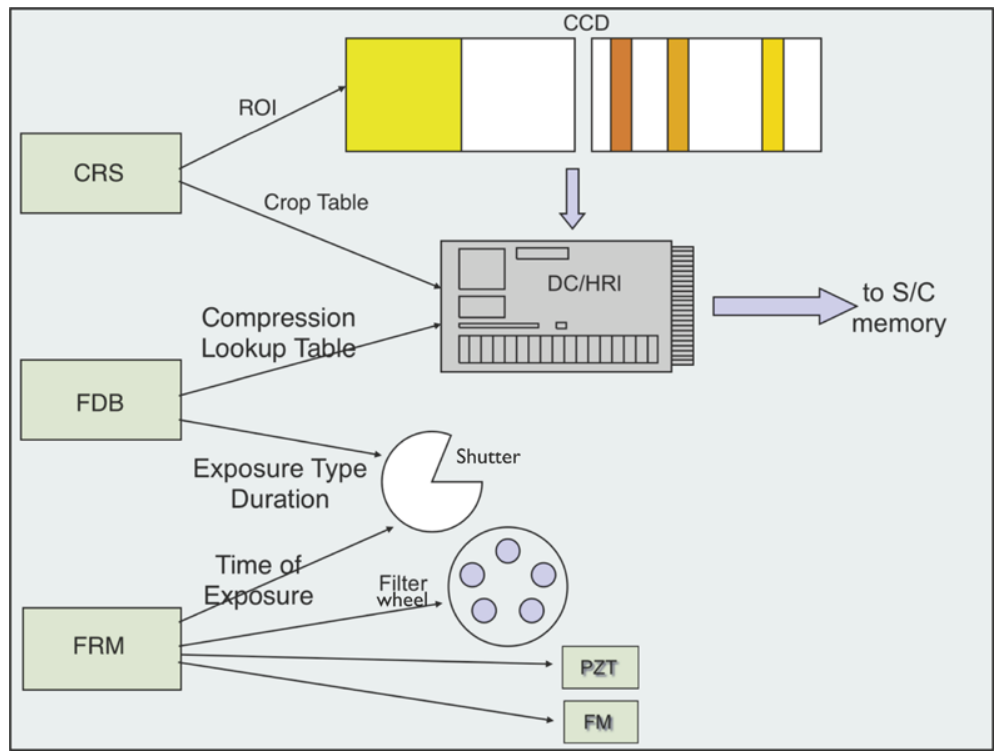

Figure 10 How IRIS images and spectra are taken and controlled by the Camera Readout Structure (CRS), Frame Definition Block (FDB), and FrameList (FRM).

ii) The FDB configures the shutter to obtain the desired exposure time.

iii) At the time specified by the FRM and OBS, the following sequence is started:

- the mechanisms are moved (if required/desired): focus position, PZT offsets, filter wheel,

- the shutter is opened,

- after exposure time, the shutter is closed,

- data are then read from the CCDs by the cameras and sent to the DC/HRI board,

- the compression parameters in the FDB are used by the DC/HRI to compress the images before sending them to spacecraft memory.

The flight software ensures that the shutters are not opened until all mechanisms have stopped moving and settled. In addition, the exposures controlled by all three shutters are synchronized so that they all end at the same time, followed by the camera readout. Depending on a parameter set in the OBS tables, the camera readout can be done in parallel for both cameras, or sequentially (thus reducing low-level noise cause by electronic interference from simultaneous readouts). The typical sequence of events is illustrated in Figure 10.

The CEBs allow for onboard summing, i.e. before digitization in the $\mathrm{A} / \mathrm{D}$ converter. This is controlled by the CRS tables, which allow any combination of $1 \times, 2 \times, 4 \times, 8 \times$ spectral summing and $1 \times, 2 \times, 4 \times$ spatial summing. The sequencer software also allows the readout of small regions of interest on any of the CCDs. This speeds up readout of the CCDs and reduces the data volume that is downlinked. The latter is also accomplished by onboard compression. IRIS follows the same compression approach as AIA. It has one data compression/high-rate interface (DC/HRI) card that performs data compression in hardware and then transmits the compressed data to the spacecraft interface and the solid-state memory board (SSMB, which has a capacity of 48 Gbits). The primary algorithm is lossless Rice compression, which typically achieves a factor of two reduction in data volume for UV spectra and images. A second, lossy algorithm is based on a look-up table, which is configurable 


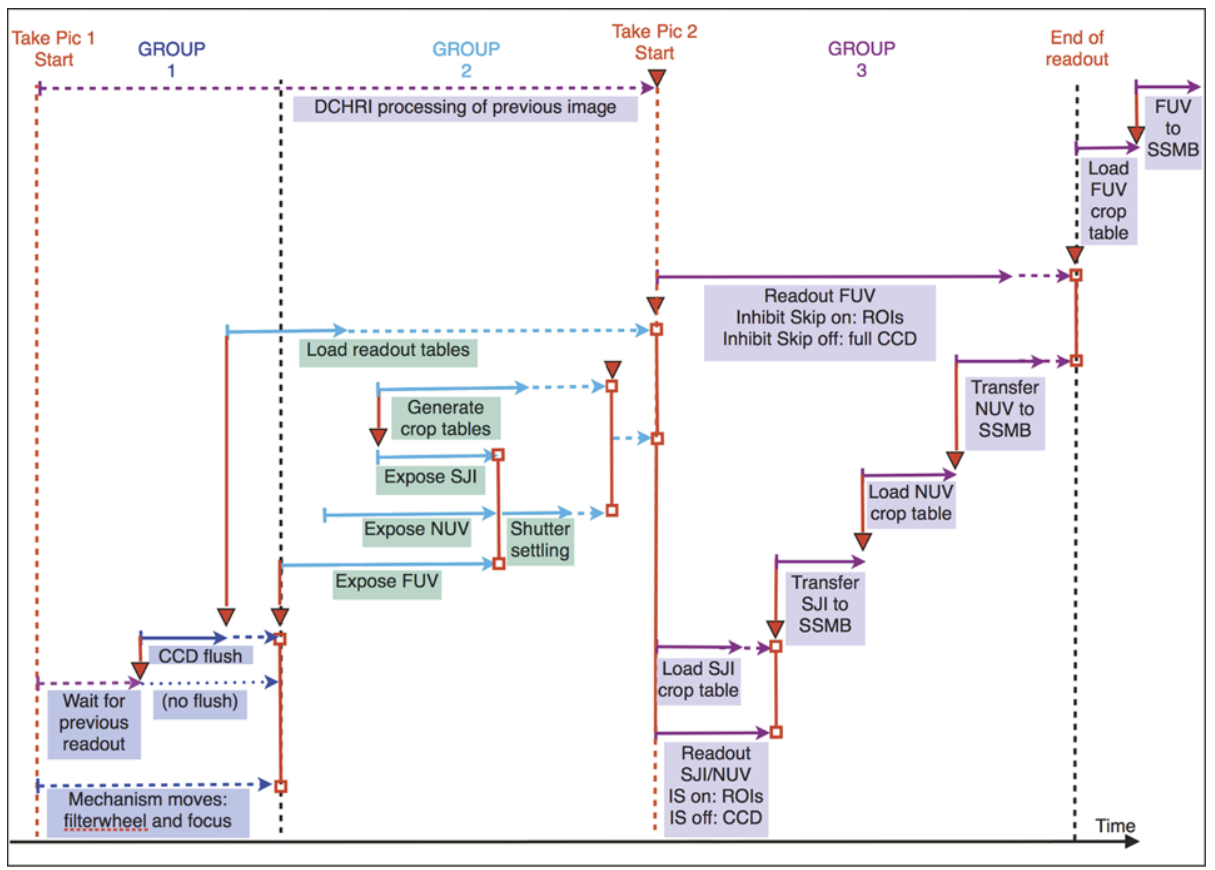

Figure 11 The sequence of events involved in taking and processing a "picture".

from the ground. The baseline tables are variations of scaled square-root function, similar to those used for HMI and AIA. This reduces the data volume by another factor of about two, depending on the type of images/spectra to which it is applied.

\subsection{Timing}

The IRIS sequencer software has been designed to take spectra and images at a very high cadence to accommodate the science goals of IRIS, which call for two-second spectral and five-second imaging cadence. The "Take Picture" command in the flight software accomplishes the key operations that enable the appropriate IRIS data to be acquired and is illustrated in Figure 11. Each activity requires a certain minimum amount of time, the timing of which defines the minimum (or fastest) cadence that IRIS can obtain. There are three groups of events that are optimized for fastest-cadence operations, and they are in part sequential and in part parallel (Figure 11):

i) mechanism moves, waiting for readout of previous image(s) and CCD flush,

ii) starts with the longest exposure time and ends with the start of the readout and/or DC/HRI processing of the previous image,

iii) camera readout and processing of the images in the DC/HRI. As soon as the readout starts, the mechanism moves and the next "Take Picture" sequence can start.

To optimize the timing of observing sequences, a ground-based "Table Creator" tool has been developed that simulates the behavior of sequencer software and hardware and adjusts the timing in the OBS and FRM tables so the instrument runs as fast as possible. 


\subsection{Default Tables}

To minimize complexity of table management and testing, as well as operations, a large number of sequences have been calculated and are available to science planners. The numbering scheme of the default OBS tables reflects their scientific goal as illustrated in Tables 12, 13, and 14. There are about 50 basic observing modes (Table 12), which vary from dense, coarse, and sparse rasters to sit-and-stare sequences of varying sizes and field-of-view choices. These basic sequences are also available with variations in SJI cadence and wavelength selection, exposure times, wavelength coverage, etc. These are listed in Tables 13 and 14. The numbering scheme is such that the same basic observing-mode properties are maintained in the last two digits of the OBS-ID, whereas leading digits indicate various choices for exposure times, SJI cadence, etc. The numbering scheme thus acts similarly to a binary mask with the digits listed in Tables 13 and 14 acting as "bits" switching options on and off. For example, OBS Table 20113644 defines a large $(126 \times 120$ arcsec $)$ raster with coarse (two arcsec) steps, a medium line-list with lossy compression, 30-second SJI images in Si IV and with two-by-two spatial binning and $2 \times$ spectral binning. Note that the leading two digits of any OBS-ID are the "version number" (not shown in the example), e.g. 3820113644 is part of table version 3.8. OBS-IDs starting with " 42 " are calibration sequences.

The basic raster modes include

- dense rasters: step size of the raster is equal to the slit width ( $0.33 \operatorname{arcsec})$.

- sparse or coarse rasters: step size of each raster location is larger than the slit width. Allows rapid scans of much larger areas, e.g. for flare or CME watch programs.

- sit-and-stare (fixed-slit mode): no rastering.

- multi-point dense/sparse rasters: involves rasters with a small number of dwelling locations to study propagation of waves, etc.

The smallest possible step size is 0.054 arcsec, which is also the granularity with which solar rotation is tracked with IRIS.

\section{Operations}

\subsection{Overview}

The IRIS polar, Sun-synchronous orbit is similar to that of TRACE and Hinode and allows for seven - eight months of continuous observations per year, with strong atmospheric absorption occurring during the November-February time frame when the Sun is seen by IRIS at heights below $\approx 200 \mathrm{~km}(\mathrm{FUV})$ and $\approx 50 \mathrm{~km}(\mathrm{NUV})$ above the Earth's surface. IRIS data are downlinked to X-band antennas in Svalbard, Norway $\left(\approx 8-9\right.$ passes day $\left.{ }^{-1}\right)$, Alaska $\left(\approx 3-4\right.$ passes day $\left.{ }^{-1}\right)$ and Wallops $\left(\approx 1-2\right.$ passes day $\left.{ }^{-1}\right)$. As a result, IRIS has an average data rate of $0.7 \mathrm{Mbits}^{-1}$, i.e. about $60 \mathrm{Gbits} \mathrm{day}^{-1}$ (compressed). Data (nominally 14 bits pixel $^{-1}$ ) are compressed onboard using Rice and lossy compression, nominally to about 3-4 bits pixel ${ }^{-1}$. Onboard memory is $48 \mathrm{Gbits,} \mathrm{allowing} \mathrm{storage} \mathrm{of} \mathrm{more}$ data-intensive observing sequences that can be downlinked later over the course of several orbits.

The high data rate, short exposure times, and flexible rastering schemes allow rapid scans of small regions on the Sun at very high spatial resolution on the order of $0.33-0.4 \operatorname{arcsec}$. 
The baseline cadence is five seconds for slit-jaw images and three seconds for six spectral windows of strong, bright lines.

IRIS operations are similar to TRACE and Hinode, with observing programs uploaded five times per week and data made publicly available within a few days of the observations. Coordination with Hinode, SDO, and a variety of ground-based observatories is a high priority.

\subsection{Timeline}

IRIS operations are controlled by a timeline that contains a list of spacecraft and instrument commands. The timeline is used by a science planner to perform the following operations:

- point the telescope to a position on (or off) the solar disk within 21 arcminutes of disk center.

- switch solar tracking on or off. When solar-rotation tracking is on, the pointing of the telescope is continuously adjusted to compensate for the rotation of the Sun so that the same region on the Sun is kept within the field of view.

- correct the pointing of the telescope to compensate for orbital wobble introduced by thermal flexing between the guide telescope and the main IRIS telescope. This is done by using an orbital-wobble table (Section 4.5).

- roll the IRIS telescope from its nominal direction (slit parallel with the N-S direction on the Sun) by an angle between -90 and +90 degrees from solar North.

- perform an upload of OBS and dependent tables.

- execute (start and stop) a number of previously uploaded OBS tables.

- set the global automatic exposure control (AEC) parameters.

- switch on and off transmission of data through an X-band downlink.

- close or open the ISS loop (to remove jitter).

The timeline is generated using the timeline tool, which is based on the TRACE timeline tool. Timelines are uploaded to IRIS every weekday. The Monday-through-Thursday timelines cover a planning period of one full day from 04:00 UT to 04:00 UT. The Friday timeline covers a time period from 04:00 UT Saturday to 04:00 UT Tuesday.

\subsection{Roll Restrictions}

The nominal direction of the IRIS slit is parallel with the solar north-south direction (roll angle $=0^{\circ}$ ). However, IRIS is capable of operating with the spacecraft rolled at any angle between $\pm 90^{\circ}$ from solar North. This enables observing programs in which the slit is parallel with the solar limb at any position along the limb (from the equator to the poles).

Operations under rolled conditions have two operational impacts or restrictions:

- reduced downlink data rate, caused by the directional X-band antenna no longer pointing "straight down to Earth" for non-zero roll angles. Some roll angles will lead to significant reduction of positive link margin and result in shorter downlink passes.

- certain roll angles are forbidden twice per month (first and last quarter of the Moon). The IRIS attitude is controlled by two star trackers at opposite ends of the spacecraft. During two periods around the first and last quarter of the Moon there are certain roll angles for which the Earth is in one star tracker and the Moon is in the other star tracker. When 
Table 6 IRIS automatic exposure control global parameters.

\begin{tabular}{lll}
\hline Name & Acr. & Definition \\
\hline Flare Flag & FF & $\begin{array}{l}\text { Controls whether IRIS goes into flare AEC mode or not. If FF = 1, SJI } \\
\text { images control exposure times of spectra } \\
\text { Controls whether the AEC software is used to change exposure times } \\
\text { AEC Flag }\end{array}$ \\
AEC Lapse Time & ALT & $\begin{array}{l}\text { Exposure times are set to default in the FDB if last SJI was taken more } \\
\text { than ALT seconds ago }\end{array}$ \\
Event Flag & EF & $\begin{array}{l}\text { Keeps track of whether flaring event has occurred or not } \\
\text { After ELT seconds, the event flag is set back to zero }\end{array}$ \\
Event Lapse Time & ELT & $\begin{array}{l}\text { Sets which of 16 AEC tables is used to determine whether an event has } \\
\text { occurred }\end{array}$ \\
\hline
\end{tabular}

that occurs the star tracker CCDs may become saturated and attitude control is no longer possible, which causes IRIS to automatically transition into a safe "non-science-gathering mode." To avoid this, certain roll angles are excluded twice a month.

\subsection{AEC Operations}

One of the science goals of IRIS is to study how flares and CMEs are triggered (e.g. by flux emergence). Obtaining spectra and images during the impulsive phase of flares is an important aspect of these types of studies. The occurrence of flares presents a major challenge for a slit-based spectrograph: intensities of spectra vary strongly spatially and temporally, which can lead to overexposure and saturation of some of the spectra. Automatic exposure control (AEC) based on spectra taken as part of a raster scan across the solar disk is not an efficient way of avoiding overexposure because the AEC input data lags behind the rapidly evolving brightness in a flare environment in time and space. The AEC approach for IRIS circumvents this problem by having the AEC for all detectors (spectra and images) be based on intensities measured in the SJI images. By design, IRIS does not switch to a different, specific observing program after flares are detected, so the current observing program will continue to run, but with SJI-based AEC for spectra and images.

The general IRIS AEC philosophy is not focused on trying to capture very weak signals. Instead it is similar to that of SDO/AIA, which is based on making sure that overexposed data do not occur too often. In other words, the AEC is used for preventing saturation during flares.

Most of the AEC operations are set at the FRM and FDB level. By default the IRIS timeline will set the AEC flag to zero, i.e. disable AEC operations (see Table 6). If the planner sets AEC to one for an observing program, the onboard software will automatically determine for each SJI image set in the Event Enable Mask (EEM) whether a flaring event (Event Flag =1) has occurred or not. The EF will be set to one by the onboard software when the computed AEC exposure times drop below a specified threshold exposure time. This threshold exposure time is set in the AEC tables. When EF is set to one, AEC of spectra and images exposure times are controlled by the AEC options listed in the FRM list. After $\mathrm{EF}$ was set to one, the instrument software sets $\mathrm{EF}=0$ when a flare condition has not been satisfied for the time specified by ELT. Similarly, AEC operations are suspended if no SJI images were taken within ALT seconds. 


\section{Calibration}

\subsection{CCD Characterization}

The four CCDs are read out by two different CEBs. The gain for the FUV CEB has been set high to six electrons $\mathrm{DN}^{-1}$. All SJI channels and the NUV use a lower gain setting of 18 electrons $\mathrm{DN}^{-1}$. All NUV photons create one electron-hole pair on the detector, i.e. one electron photon ${ }^{-1}$. This means that for NUV spectra and NUV SJI channels 2796 and 2832 there are about 18 photons $\mathrm{DN}^{-1}$. For FUV photons about 1.5 electrons photon ${ }^{-1}$ are created on average. This means that for FUV spectra there are about four photons $\mathrm{DN}^{-1}$, while for FUV SJI channels 1330 and 1400 there are about 12 photons $\mathrm{DN}^{-1}$.

The dark frame levels for IRIS have two components: a pedestal level $[P]$ set electronically, and the dark current. Each CCD read port exhibits slightly different behavior. Extensive tests pre- and post-launch have led to the following model for the total dark level $[D]$ in read port $j$ :

$$
D_{j}=P_{j}\left[T_{\mathrm{CEB} j}\left(t-\delta t_{j}\right)\right]+\mathrm{e}^{\left(a_{j}+b_{j} T_{\mathrm{CCD} j}\right)} n_{x} n_{y} t_{\text {int }}+\Delta D_{j}\left(x, n_{x}, n_{y}, t_{\text {int }}\right) .
$$

Here, $P_{j}$ is the pedestal level in read port $j$, a function of CEB temperature $\left[T_{\mathrm{CEB} j}\right]$ for that port, time lagged by $\delta_{t}$. The second term models the average dark-current rate, which is the product of an exponential dependence on the CCD temperature $\left[T_{\mathrm{CCD} j}\right]$, the amount of on-chip summing $\left[n_{x} n_{y}\right]$, and the time between $\mathrm{CCD}$ reads (i.e. the dark-current integration time) $\left[t_{\text {int }}\right]$. A final term $[\Delta D]$ models the change in shape of the dark in the wavelength $[x]$ direction as $t_{\text {int }}$ and summing are increased, from flat for $t_{\text {int }} \approx 0$ seconds and $1 \times 1$ summing, to roughly bilinear in $x$, sloping gradually and then more rapidly away from the read-out point, with an end-to-end amplitude of $\Delta D \approx 10 \mathrm{DN}$ for $n_{x} n_{y}=32$ and FUV ports.

In practice, the $D_{j}$ function is computed for each port and added to the appropriate portion of an averaged, cleaned "basal" dark. This basal dark was constructed from 19 $t_{\text {int }} \approx 0$ seconds images, which had particle hits and (some) hot pixels removed; the average value in each read-port zone was then set to zero. Analysis of 152 full-frame $1 \times 1$ darks indicates average errors at $t_{\text {int }}=30$ seconds of $<0.12 \pm 0.12 \mathrm{DN}$ in the zero point (worst case) for the FUV ports, and $<0.08 \pm 0.03 \mathrm{DN}$ for the NUV/SJI ports. Scatter in the residual RMS (dark - model dark) is $<3.1 \mathrm{DN}$ (FUV ports) and $<1.2 \mathrm{DN}$ (NUV/SJI), and essentially reflects the camera readout noise. Results deteriorate somewhat as summing increases: for $n_{x} n_{y}=8$, average offsets are $<1.6 \pm 2.5 \mathrm{DN}$ for FUV, and $<0.16 \pm 0.82 \mathrm{DN}$ (NUV/SJI), with RMS residuals of $<3.3 \mathrm{DN}$ (FUV) and $<1.2 \mathrm{DN}$ (NUV/SJI). These calibrations will be revisited and refined periodically as more data are taken and more operational temperatures are sampled over the course of the spacecraft's thermal "year".

On orbit, it was discovered that when both CEBs are read out simultaneously, a readinterference noise pattern is superimposed on the resulting data. While it does not always occupy the entire read-port zone spatially, the pattern, where it is present, is approximately constant in the spatial direction for a given readout and read port. The portions of the CCD showing the pattern show mirror symmetry across the two read-port zones of a given chip, and both CCDs show identical coverage. The pattern's wavelength structure is complex and varies in shape and period from readout to readout, with a typical period of about 340 pixels $(1 \times 1$ summing). The period is constant for all ports of a given readout, though its shape varies from port to port. One port typically has the highest amplitude pattern (in FUV $\mathrm{SG} \approx \pm 3 \mathrm{DN}$ ), with other ports showing decreasing amplitudes by successive factors of $\approx$ two, and simpler wavelength structure. This pattern is barely visible in the high-contrast, high-S/N NUV spectra and all SJI images. It is more apparent in FUV spectra, which have 
higher gain and typically have lower counts. To mitigate this pattern noise, IRIS planners can choose to use non-simultaneous reads, which eliminates the pattern completely at the cost of lower cadence. The IRIS team is also working on software to model and remove the readout pattern.

\subsection{Flat Field}

Flat-fielding of images acquired with IRIS CCDs is required to remove the effects of

- vignetting,

- the semi-regular laser anneal pattern visible at the 5-10\% level in CCD images,

- potential dose accumulation burn-in,

- dust and artifacts on the slit and detector,

- the intensity pattern due to irregularities in the slit width.

Flat fields for IRIS are constructed from solar observations for the NUV and FUV spectrographs and the slit-jaw imagers with the same intent, to extract structure due to the instrument from structure due to the Sun, although two different approaches are used.

\subsubsection{Strategy for the Spectrograph Flats}

The spectrograph flat fields are produced by extracting the spectral profile from spectrograph images that have been sufficiently averaged. The emission lines of the FUV and the $\mathrm{Mg}$ II line cores show high-contrast features and non-uniform line profiles, even in the quiet Sun. A spectrum that is spatially smooth and spectrally uniform is obtained by taking a coarse raster of the disk-center quiet Sun, during which the telescope is de-focused and slewed during the exposures to provide additional smoothing, then the images in the sequence are averaged together. At least 200 images are required to build a good intermediate flat field.

To extract the spectral profile the intermediate flat field is up-sampled by padding the Fourier transform of the image. This image is warped using the geometry correction determined for each spectral window to make the spectral and fiducial lines straight with respect to the grid of pixels. Averages are taken along the spatial and spectral dimension. These averages are reversed through the geometry correction, re-sampled back down to the original image size, and divided from the original spectrum to produce the final flat field. The spatial flat field (average along the spectral dimension) is reserved so that it can be shifted and applied to properly compensate for slight thermal shifts of the spectrum on the detector.

Exposure times of 15 seconds are used for each individual image for both the NUV and FUV flat-field observations. For the FUV spectra, this ensures that the bright lines have enough signal, but the faint lines and continuum do not rise above the level of the read noise.

Analysis of the FUV spectra has revealed a faint, smooth, stray-light background that needs to be removed before flat-fielding. This background does not contain the CCD annealing pattern that is present at blue and UV wavelengths, indicating that it is a visible-light leak, whose photons penetrate more deeply into the CCD. A gain of 5.6 \pm 0.2 photons $\mathrm{DN}^{-1}$ has been measured for the stray-light background in long exposures taken off the solar limb, which is comparable with the gain measured with the blue LEDs and consistent with the statistics expected for visible photons. Analysis of the off-limb data from the other channels of IRIS does not indicate a similar stray-light component. The FUV background is on the order of $0.5 \mathrm{DN} \mathrm{s}^{-1}$ at most and has been found to drop with distance from Sun center to about $0.15 \mathrm{DN} \mathrm{s}^{-1}$. This drop-off varies with position angle of the slit relative to the Sun, 
Table 7 Best focus position (in steps) for each IRIS science channel.

\begin{tabular}{lc}
\hline Channel & Focus position \\
\hline SJI $1330 \AA$ & -114.4 \\
SJI $1400 \AA$ & -114.5 \\
SJI $2796 \AA$ & -113.6 \\
SJI $2832 \AA$ & -111.8 \\
NUV SG & -116.3 \\
FUV SG & $\approx-115$
\end{tabular}

which suggests that the background does not enter through the slit. It is more likely due to a low-level light leak in the labyrinth structure of the slit-prism mount. The intensity of the background also depends on the position of the SJI filter wheel. It is slightly higher with an NUV filter in place, probably because these filters reflect some visible light back to the slit prism area. The background has been characterized and is being removed before the FUV spectrograph flat fields are applied in our Level 1.5 calibration.

\subsubsection{Strategy for the Slit-Jaw Imagers}

Flat fields for the slit-jaw imagers are constructed using the technique presented by Chae (2004), which makes use of relatively shifted solar images to extract the stationary flat-field pattern of the instrument from the moving solar scene. For this implementation we have selected a Reuleaux triangle dither pattern of $20 \operatorname{arcsec}$ that contains 15 pointings. This way the dither can be accomplished on the short timescale of the solar chromosphere. The results of multiple pointings are combined to produce the final flat field. Regions of bright, but quiet, plage provide sufficient counts for the FUV. For the filters with high contrast (FUV and NUV core), images are taken with the telescope defocused so that a more uniform coverage of the CCD is possible, while images for the lower-contrast NUV wing and glass filters are taken in-focus to ensure the granulation pattern of the quiet Sun can be accurately extracted.

The Chae method significantly decreases the amount of time spent obtaining flat-field images. Each wavelength of the SJI uses different optics, causing a change in the vignette pattern. In addition, the CCD has a slightly different gain response at each wavelength, necessitating the use of separate flat fields for each filter. Using the Chae method, only $15 \times 3$ flats are necessary for each filter, whereas an averaging method might take hundreds of images to sufficiently smooth over the solar structure.

The fiducial marks in the spectra and the slit in the SJI images are important for calibration and are therefore seamlessly removed from the flat field by replacing the pixels around each with the pre-launch flat-field images obtained using a mercury lamp filtered to $2537 \AA$.

Flat-field calibration data will be acquired regularly to track changes (e.g. during eclipse season, or because of burn-in) with appropriate changes made to the calibrated Level 2 data.

\subsection{Optical Performance}

\subsubsection{Focus}

Focus is adjustable by moving the telescope secondary mirror in $2.28 \mu \mathrm{m}$ increments, over a range from -210 to +350 steps (a full range of $1277 \mu \mathrm{m}$ ). The best position of telescope focus was determined by analyzing multiple sequences of solar images at different focus positions. The results are summarized in Table 7. Uncertainties are smaller than one step, 

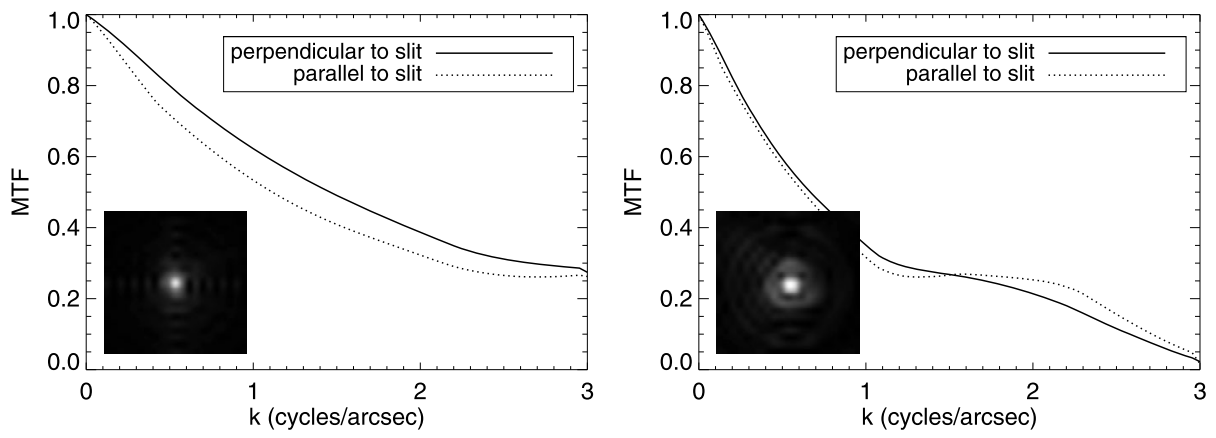

Figure 12 MTF of the IRIS FUV (left) and NUV (right) SJI, derived from phase-diversity analysis of a series of exposures adjusting through focus at $1330 \AA$. The PSF, inset, has been square-root-scaled to bring out faint features.

except for the FUV spectrograph. The profusion of transition-region explosive events makes the quantitative estimate of focus difficult for the FUV, but visually it is near -115 . Depth of focus is approximately \pm 5 steps. IRIS runs routinely at the designated best instrument focus of -115 . During eclipse season, the focus changes slightly to values around -121 .

\subsubsection{Point Spread Function and Spatial Resolution}

The spatial resolution of IRIS is very good in both the spectrograph and SJI. Figure 12 shows point-spread functions and modulation-transfer functions for the FUV 1330 and NUV 2832 channels of the IRIS SJI. These were derived from on-orbit focus tests using a phasediversity code described by Löfdahl and Scharmer (1994). The code was run on a small region centered in the lower-left portion of each detector (coordinates 150, 250). The NUV

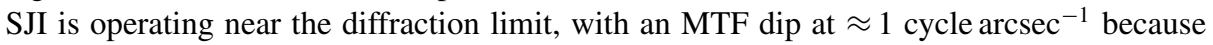
of the telescope central obscuration. The MTF falls to $10 \%$ at $2.6 \mathrm{cycles} \operatorname{arcsec}^{-1}$, which exceeds the resolution requirement for the instrument. The NUV images have a two-pixelwide core containing $\approx 42 \%$ of the light.

The figures show that FUV images are nearly pixel-limited, with the MTF above 0.25 all the way to the Nyquist frequency: the PSF core is a single bright pixel containing $34 \%$ of the light, and the $3 \times 3$ brightest pixels have $70 \%$.

\subsubsection{Spectral Resolution}

Preliminary measurements of the spectral resolution of IRIS show it to be excellent, and essentially limited by the Nyquist criterion. We analyzed quiet-Sun spectra taken on 20 August 2013 and used single Gaussian fits to the O I $1355.6 \AA$ and Mn I $2801.85 \AA$ lines to determine the FWHM of these lines for 1000 positions along the slit. We find that many profiles of these lines are very narrow with average FWHM of $25.85 \mathrm{~m} \AA$ in the FUV and $50.54 \mathrm{~m} \AA$ in the NUV, i.e. below and around the Nyquist criterion. This is illustrated in Figure 13, which shows histograms of the width measured in these neutral lines.

\subsubsection{Plate Scale}

The spatial plate scales for the SJI shown in Table 3 were determined using a meridional scan, and then assembling all of the images into a north-south mosaic across the full disk. 

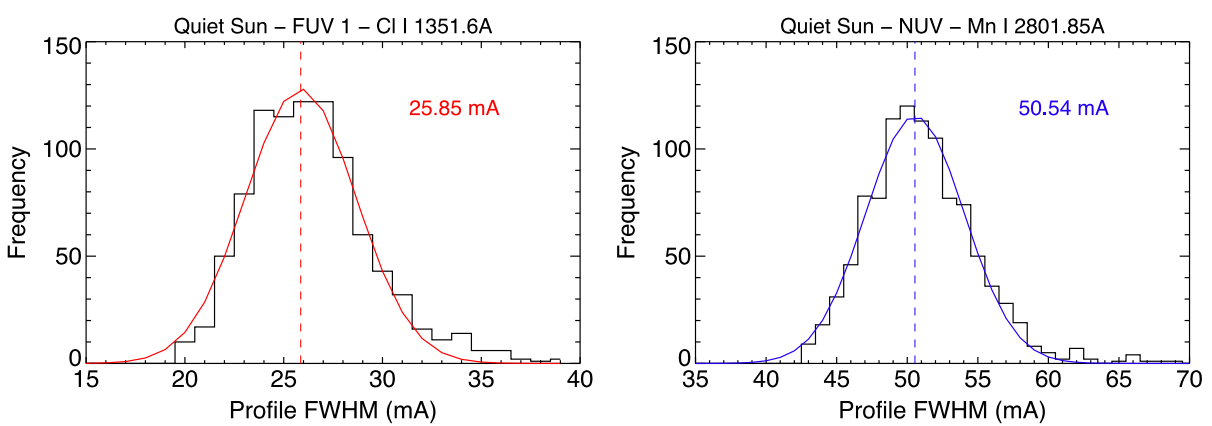

Figure 13 Histogram of the FWHM of the O I $1351.6 \AA$ and Mn I $2801.85 \AA$ lines in a quiet-Sun region indicates that the effective spectral resolution is limited by the Nyquist criterion. The dashed lines show the averages of the distributions.

In that mosaic, the solar diameter in pixels is compared with the known solar diameter, corrected for the distance to the Sun at the time of the observations. The SG plate scales (Table 2) were determined by matching the distance between the two fiducial marks on the SJI and SG data and applying the resampling factor to the previously determined SJI plate scales. Note that at data processing Level 1.5 (and higher) all data are placed onto a common plate scale of $0.16635 \mathrm{arcsec}_{\mathrm{pixel}}{ }^{-1}$.

The spectral plate scales (Table 2) are average values across the detector. These prelaunch values agree very well with the averages from post-launch calibration. Note that the actual spectral plate scale is wavelength dependent. This non-linearity in the plate scale is corrected in Level 1.5 data (and higher) where the data are interpolated onto the average plate-scale grid.

\subsection{Compression}

Compression of IRIS data for efficient transmission to the ground is accomplished through two methods: lossless Rice compression (Rice and Plaunt, 1971) and optional lossy compression using look-up tables (LUTs).

Rice compression takes advantage of the relative smoothness of data by taking a running difference and encoding values in two parts. The rapidly changing, least significant bits are represented directly and the slowly changing, most significant bits are compressed by the running difference. Data values in an image can be losslessly compressed by about a factor of two using this method. The Rice K-value selects the number of least significant bits to be directly represented. For IRIS, the K-value for a given observation is set in the FDB during the generation of observing tables. The K-value for most efficient compression depends on the structure in the image and the exposure time. The best $\mathrm{K}$-values have been determined for a variety of images and spectra.

Additional compression of the IRIS data can be achieved by applying a lossy algorithm using an LUT. For IRIS we make use of the LUTs generated for AIA and HMI data from the SDO mission. These compression tables consist of a linear ramp at low values and transitions to a square-root function at approximately the level of the read noise. This way low counts are not compressed, but higher counts are compressed more and more toward the top of the table. Compression with a square-root function introduces look-up error in a constant proportion to the photon-counting noise, resulting in a constant signal-to-noise ratio for each table. The calculated effective ratios of the LUT error to photon+read noise, based on the 
Table 8 Effective S/N Ratios for AIA and HMI LUTs.

\begin{tabular}{llll}
\hline ID & Name & FUV ratio (low gain) & NUV ratio \\
\hline 0 & No Compression & 0.0 & 0.0 \\
1 & Inverse & 0.0 & 0.0 \\
2 & hmi c3 plin & 0.28 & 0.32 \\
3 & aia csnr 025 & 0.91 & 1.0 \\
4 & aia csnr 050 & 1.8 & 2.1 \\
5 & aia csnr 100 & 3.7 & 4.2 \\
6 & hmi c3 5 plin & 0.40 & 0.46 \\
7 & hmi c4 plin & 0.57 & 0.65 \\
8 & hmi c4 5 plin & 0.81 & 0.92 \\
9 & aia csnr 200 & 7.6 & 8.6 \\
\hline
\end{tabular}

noise level expected from a given detector, are tabulated for all the LUTs available for IRIS in Table 8. The values shown are calculated for the CCD pedestal levels that were adjusted after launch.

\subsection{Pointing Stability}

Thermal flexing between the guide telescope and the main IRIS telescope introduces a wobble in the observations on timescales of an orbit (see Section 4.5). We measured this wobble on-orbit by taking SJI 2832 channel data at the solar North Pole and East Limb, at 20-second cadence, covering more than an orbit, and then running a cross-correlation algorithm on the data. To explore the effect of roll angle on the wobble, we have carried out this analysis for five roll angle values: $0^{\circ},+90^{\circ},-90^{\circ},+45^{\circ}$, and $-45^{\circ}$. The results of this analysis show that the wobble is, peak to peak within an orbit, on the order of three arcsec in the $x$-direction, and one arcsec in the $y$-direction (see Figure 14a, showing the wobble curves for roll $0^{\circ},+90^{\circ}$, and $-90^{\circ}$ ). For different roll angles we find that the wobble is of the same order of magnitude as for $0^{\circ}$ roll angle, although with a phase shift. In particular, we find that for roll angle $\alpha$, the wobble can be approximated by the wobble for $0^{\circ}$ roll angle, shifted in phase by $\alpha / 360^{\circ}$. This is illustrated in the right panel of Figure 14, where we show the wobble curves of +90 and -90 shifted in phase by +0.25 and -0.25 respectively, plotted over the wobble curves for $0^{\circ}$ roll angle.

The effect of the wobble is corrected in regular IRIS operations with orbital wobble tables (OWT), used to apply an adjustment to the pointing that compensates for the orbital wobble. We estimate that the use of the OWTs corrects most of the wobble, with a possible residual two-IRIS-pixel effect over the course of a full orbit $(\approx 97$ minutes). This residual is larger during eclipse season. For the angles used more frequently $\left(0^{\circ}, \pm 90^{\circ}, \pm 45^{\circ}\right)$ we use wobble tables derived by corresponding calibration data, as described above. However, deriving OWT for a larger set of roll angles is unreasonably time-consuming (each angle requires calibration data for two orbits, i.e. 195 minutes, for each of the two pointings), especially considering that the wobble calibrations will need to be run regularly, several times a year. Therefore, for all other roll angles, we apply the $0^{\circ}$ roll-wobble curves, shifted in phase by $\alpha / 360^{\circ}$ as described above.

The availability of a continuous full-disk archive of UV and EUV (SDO/AIA) and continuum $(\mathrm{SDO} / \mathrm{HMI})$ images at $\approx 0.5$ arcsec spatial resolution and $\approx 24$ seconds temporal resolution allows for the registration of every IRIS slit-jaw image by means of cross-correlation of each image with a field-of-view-matched, extracted sub-field from the nearest-in-time 

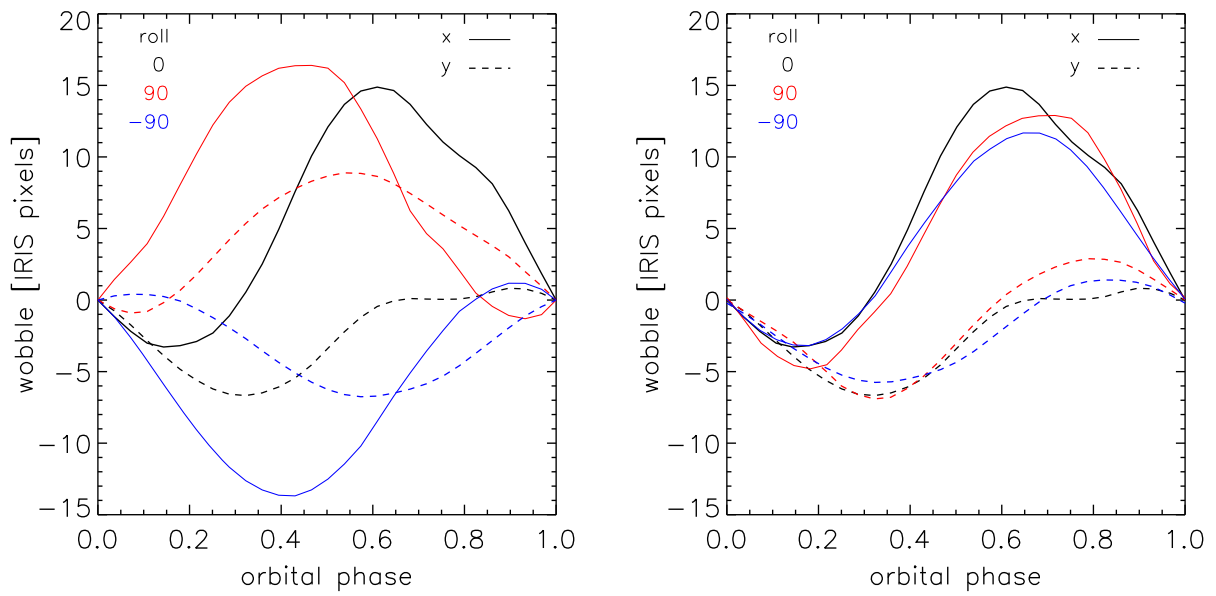

Figure 14 Orbital wobble correction. Left panel: IRIS orbital wobble in units of IRIS pixels, for the $x$ (solid lines) and $y$ (dashed lines)-directions, and three roll angle values: 0 (black), +90 (red), and - 90 (blue). Right panel: Same curves as in the left panel, but with a phase shift of the wobble curves for +90 and -90 , of +0.25 and -0.25 , respectively. The shifted curves are very similar to the wobble curves for $0^{\circ}$ roll angle. The orbital phase is zero at the time when IRIS passes through its ascending node.

SDO image of the appropriate waveband with comparable response. This technique will be used to cross-correlate IRIS 1400 slit-jaw images with AIA 1700 image sub-fields, and IRIS 2832 slit-jaw images with HMI continuum image sub-fields, to extract residual translation offsets for the IRIS slit-jaw images, which in turn can be used to update the relevant World Coordinate System (WCS) keywords in the file headers of both the slit-jaw images and spectra, and thus improve the absolute accuracy of the pointing information.

\subsection{Wavelength Calibration}

The wavelength calibration of IRIS aims to remove instrumental effects from the spectra so that these can be used to diagnose solar conditions. The calibration involves two aspects, geometric correction and relative calibration to photospheric spectral lines, which are described in the following.

\subsubsection{Geometric Correction}

As with many spectrographs, IRIS suffers from slight spectral distortion and misalignment, mainly rotation, spectral curvature, and the non-linearity of wavelength. For analysis purposes it is highly desirable to have the spectral and spatial coordinates rectilinear with the grid of pixels and to have a constant dispersion per pixel.

The geometry of IRIS is determined from heavily averaged, high signal-to-noise spectra, such as those created at the intermediate stage during the spectrograph flat fields. From these average spectra the centers of spectral lines and fiducial marks are determined by Gaussian fitting. The desired positions of the spectral lines and fiducials are calculated using the average line position at the center of the $\mathrm{CCD}$. The transformation to rectilinear coordinates is determined by fitting a two-dimensional polynomial to the measured and desired line centers. 
Once the geometric transformation is successfully determined, the solution of the wavelength can be calculated. The center positions of neutral and low-ionization lines are used to calculate the relationship between wavelength and pixels. This solution is used to modify the geometric result. An interactive tool (iris_spec_cal.pro) has been implemented in SolarSoft to make this procedure relatively simple.

The geometry correction is implemented in iris_prep.pro (SolarSoft) using cubic interpolation, which causes some smoothing of the resulting spectrum. The spatial and spectral coordinate for every pixel in the untransformed data is also available.

\subsubsection{Relative Calibration}

In addition to the geometric corrections, there are also slight thermal drifts and associated wavelength shifts. The low-Earth, Sun-synchronous orbit of IRIS creates a thermal environment in which periodic temperature variations occur within the instrument on timescales of one orbit (as well as over a year). To compensate for some of these variations, heaters are used to control some of the temperatures within the instrument. The end result is a relatively stable thermal environment that nevertheless shows some variations over an orbit.

Pre-flight thermal testing indicates that the high-order aspects of the spectral geometry do not change, but the spectral lines shift with thermal variation. These small drifts of the spectral lines on the detector are on the order of two spectral pixels, i.e. $\approx 6 \mathrm{~km} \mathrm{~s}^{-1}$. During calibration, these drifts were determined by analyzing the wavelength shifts of neutral (and Fe II) lines, formed in the photosphere or chromosphere, in the FUV 1, FUV 2, and NUV detectors. These lines, when averaged in time and space, show very little variation compared with the high velocities in the upper chromosphere and transition region, and can thus be used to determine whether wavelength shifts for the different detectors are well correlated with one another and/or with instrument temperatures.

We did not find reliable correlations between the measured temperatures and the observed wavelength shifts. Therefore, it is not possible to derive a simple relationship between changes of temperatures and line positions that can be applied to all observations. However, we found that the wavelength shifts over the course of an orbit are well correlated between FUV1, FUV2, and NUV after removal of the orbital velocity of IRIS with respect to the Sun. The wavelength shifts with time of the O I $1355.6 \AA$ line in FUV 1 , S I 1396.11 $\AA$ in FUV 2, and Ni I 2799.47 $\AA$ line in NUV are well correlated, as shown in Figure 15. The one- $\sigma$ residual of the difference between the temporal variations of NUV with those in FUV is about $0.5 \mathrm{~km} \mathrm{~s}^{-1}$. This is much lower than the typical TR velocity $\left[\approx 5 \mathrm{~km} \mathrm{~s}^{-1}\right]$. These correlations are found to hold for different roll angles of the spacecraft [ \pm 90 degrees].

Using these results, the IRIS wavelength-calibration approach is to determine the wavelength shifts of the Ni I $2799.474 \AA$ line for every NUV spectrum taken. This line has significantly higher $\mathrm{S} / \mathrm{N}$ than the FUV neutral lines. We average these shifts along the slit to determine the average thermal drift of the line, and then use this to establish a wavelength calibration of not only the NUV passband, but also the FUV 1 and FUV 2 (using the correlations discussed above).

Because the intrinsic average velocity exhibited by these photospheric neutral lines is lower than $0.5 \mathrm{~km} \mathrm{~s}^{-1}$ (based on MHD simulations), our calibration leads to an absolute calibration of the NUV passband with a precision of $\approx 1 \mathrm{~km} \mathrm{~s}^{-1}$. 

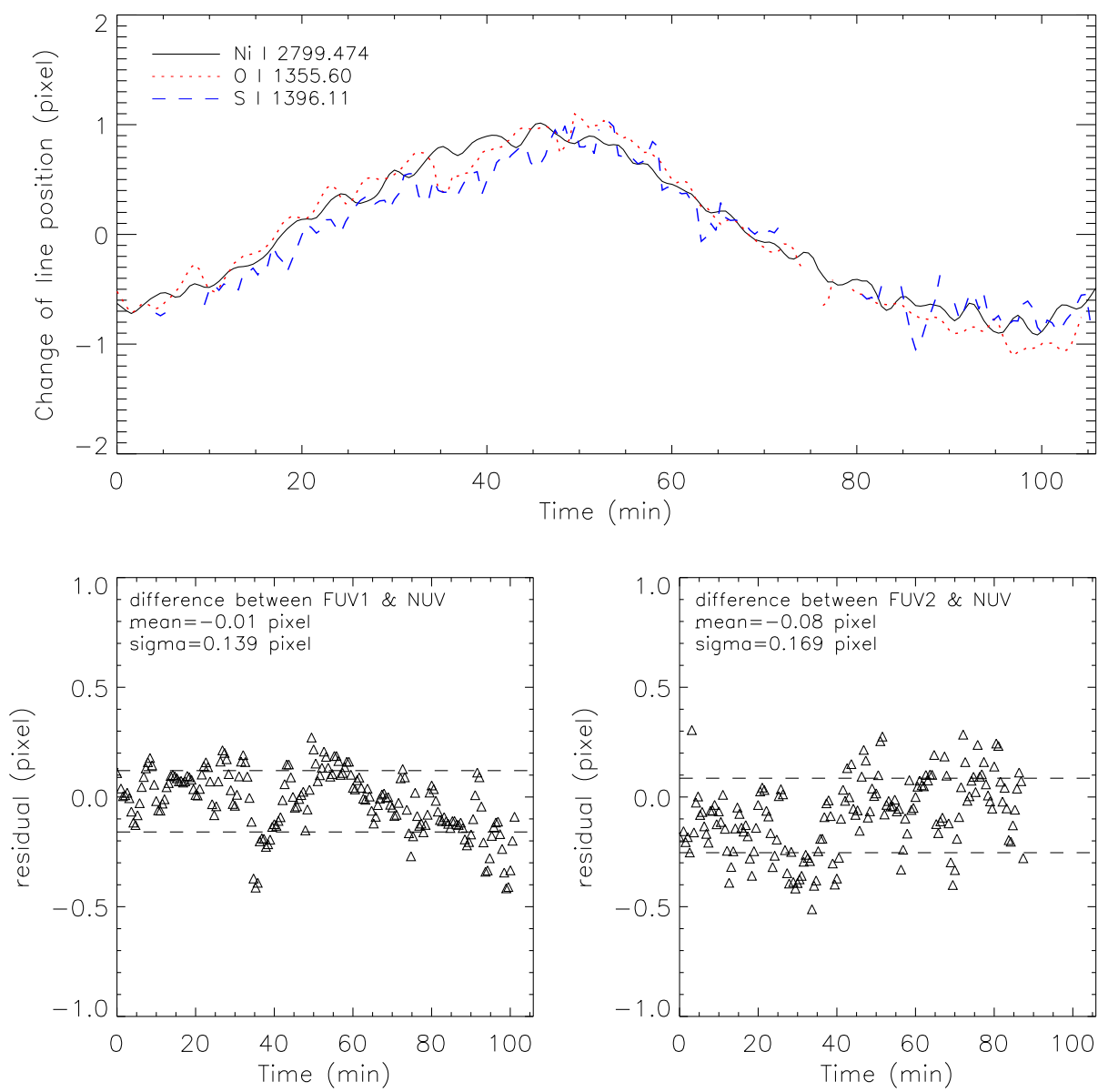

Figure 15 Wavelength-calibration approach. Top panel: correlation between wavelength shifts of neutral lines in FUV 1 ( O I 1355.6 ̊), FUV 2 (S I 1396.1 $)$ ), and NUV (Ni I $2799.5 \AA$ ) as a function of time during one orbit. Bottom panels: differences of wavelength shifts between FUV 1 and NUV (left panel) and between FUV 2 and NUV (right panel).

\subsection{Throughput}

\subsubsection{FUV Passbands}

IRIS's off-limb capability and high sensitivity allow us to use UV-bright stars as calibration sources. We have identified about a dozen stars that should be detectable by IRIS. In August 2013, we demonstrated our stellar calibration capability with early observations of HD 86360 and HD 91316. Stellar calibration allows us to convert IRIS fluxes into physical units and to measure, on-orbit, the effective area of the SJIs and spectrograph. By observing the same stars throughout the mission, we will have an excellent measure of the change in throughput due to contamination and degradation.

The August 2013 campaign included specifically designed observing programs for two UV-bright stars: HD 86360 and HD 91316. We successfully observed both stars in the SJI 
Table 9 Effective area for each IRIS science channel.

\begin{tabular}{llll}
\hline Channel & $\begin{array}{l}\text { Effective Area }\left[\mathrm{cm}^{2}\right] \\
\text { Pre-launch }\end{array}$ & $\begin{array}{l}\text { Effective Area }\left[\mathrm{cm}^{2}\right] \\
\text { Flight }\end{array}$ & $\begin{array}{l}\text { Radiometric conversion } \\
{\left[\left(\mathrm{erg} \mathrm{s}^{-1} \mathrm{sr}^{-1} \mathrm{~cm}^{-2} \AA^{-1}\right) /\left(\mathrm{DN} \mathrm{s}^{-1}\right)\right]}\end{array}$ \\
\hline SJI 1330 & 0.5 & 0.41 & N/A \\
SJI 1400 & 0.6 & 0.81 & N/A \\
SJI 2796 $\AA$ & 0.005 & N/A & N/A \\
SJI 2832 & 0.004 & 0.0043 & N/A \\
NUV SG & 0.2 & N/A & N/A \\
FUV 1 SG & 1.6 & 1.2 & 2960 \\
FUV 2 SG & 2.2 & 2.2 & 1600 \\
\hline
\end{tabular}

1400 and 1330 filters. By changing pointings and slit locations with time, the FUV spectrum of HD 91316 was successfully recorded at about 20 locations of the slit.

We evaluated the effective areas for both SJI and SG, and performed radiometric calibration for the FUV spectra of IRIS. Spectra of both stars were observed by the International Ultraviolet Explorer (IUE) about 30 years ago. The IUE spectral radiances are given with uncertainties of $10-15 \%$ (one $\sigma$ ). Implicit in cross calibration with stars is the assumption that the irradiances of the stars are nearly constant in time, which is the case for both of these stars.

We evaluated the effective areas of the SJI 1400 and 1330 bandpasses using data for both stars. The average effective area is about $35 \%$ larger than the pre-flight value for the SJI 1400 filter and $12 \%$ smaller than the pre-flight value for the SJI 1330 filter.

Using data from the star HD 91316, we evaluated the effective areas of the two FUV wavelength bands. The average effective area is about $1.2 \mathrm{~cm}^{2}$ for FUV 1 and $2.2 \mathrm{~cm}^{2}$ for FUV 2. Both are within the error margin of the pre-flight estimates.

We performed radiometric calibration of the FUV spectra of IRIS based on the HD 91316 observation. By comparing the calibrated IUE flux with the uncalibrated IRIS spectrum of the star, we derived the radiometric calibration factor for IRIS. This factor is about 2960 in the FUV 1 band and 1600 in the FUV 2 band. Using this factor, we can convert the observed count rate $\left[\mathrm{DN} \mathrm{s}^{-1}\right]$ into radiance of the spectrum $\left[\mathrm{erg} \mathrm{s}^{-1} \mathrm{sr}^{-1} \mathrm{~cm}^{-2} \AA^{-1}\right]$. These values are summarized in Table 9.

\subsubsection{NUV Passbands}

The stars that we observed are not bright enough in the NUV. For the calibration of the NUV channels, we compared IRIS quiet-Sun disk-center observations on 19 July 2013 with fullSun spectra of 15 July 2013 taken with the Solar Stellar Irradiance Comparison Experiment (SOLSTICE) onboard the Solar Stellar Irradiance Comparison Experiment (SORCE). We scaled the IRIS data to full disk, and applied a limb darkening correction $F_{\text {tot }} / I_{\text {ctr }}=0.588$ at $2800 \AA$ (Allen, 1964). The SOLSTICE spectrum was then folded with the IRIS pre-launch effective area. A comparison of the spectra shows a good match in the wings of the $\mathrm{Mg}$ II $\mathrm{h}$ and $\mathrm{k}$ lines, with discrepancies near the line core. The latter is to be expected since the line cores are affected by solar activity: the SOLSTICE spectrum includes all active regions on the disk, while IRIS only observed at the quiet-Sun near disk center. For the wing of the lines, we can nevertheless determine how well our pre-flight effective area values for the NUV 2832 SJI channel (in the wings of $\mathrm{Mg}$ II h/k) compare with the flight values. We found that the flight values are $8 \%$ higher than the pre-flight values, i.e. within the uncertainty of the calibration method. 
Table 10 IRIS data levels.

\begin{tabular}{ll}
\hline Level & Description \\
\hline 0 & depacketized, raw images with housekeeping and overscans \\
1 & reorient images to common axes: North "up" $\left(0^{\circ}\right.$ roll $)$ increasing $\lambda / x$ to right \\
1.5 & dark current and offsets removed \\
& flag bad pixels and spikes pixels \\
& flat-field correction \\
& geometric and wavelength calibration \\
& physical units (exposure and photon conversion) \\
& recast as rasters and SJI time series, standard science product \\
3 & recast as 4D cubes for NUV/FUV and 3D cubes for SJI for CRISPEX \\
HCR & description of observing sequences, ingested by HCR at LMSAL \\
\hline
\end{tabular}

The NUV 2796 SJI channel and the NUV spectrograph pre-flight values are given in Table 9.

\subsubsection{Temporal Evolution}

To determine the long-term evolution of the sensitivity of the various IRIS passbands, we will continue to perform stellar calibrations as the targets appear in the IRIS field of view. This will help calibrate any long-term trends of the sensitivity of the FUV channels. In addition, IRIS obtains observations of a quiet region near disk center on a daily basis to monitor the throughput of both FUV and NUV channels.

\section{Data Processing}

\subsection{Level 0 to 1.5}

IRIS data-processing leverages the heritage infrastructure developed for SDO and Hinode. Raw science data flow from the Mission Operation Center (MOC) at NASA/Ames to the Joint Science Operations Center (JSOC) at Stanford University and LMSAL. The IRIS data system uses the same infrastructure as that for SDO/AIA and HMI. At the JSOC, the science telemetry is archived, with a copy exported to the backup site at LMSAL. After receipt from the MOC, the telemetry is converted into Level 0 image files (see Table 10). To generate Level 1 data, mirroring of various axes is removed and various headers are added that contain information about temperatures, roll, and pointing. It is automatically produced in a pipeline at the JSOC. All these data levels are stored within the JSOC data system.

Level 1.5 is produced at LMSAL and is the lowest level of scientifically useful data. Dark current and systematic (pedestal) offsets are removed, and flat-fielding corrections for telescope and CCD properties are applied, as well as geometric and wavelength corrections. All images are mapped to a common spatial plate scale. Spectral images are also remapped to align with an equal-sized array where wavelength and spatial coordinates align with the grid. An array mapping the wavelength axis to physical wavelength is created in this process. As with AIA, equivalent procedures to those used internally to transform Level 1 to Level 1.5 are distributed via SolarSoft as iris_prep.pro. 
Level 1 and 1.5 data products are still organized per "CCD frame", i.e. every exposure taken by IRIS is stored as a FITS file. The format of these files is compressed FITS files, similar to AIA and HMI: full frames are stored even when only some regions of interest (ROI) were obtained during a particular exposure. In this case, most of the FITS array is set to a "Not a Number" (NaN), with only the ROI displaying observed data numbers.

\subsection{Level 2 and 3}

Level 2 is generated at LMSAL using Level 1.5 data. Level 3 data can be produced by the user with iris_make_fits_level3.pro (SolarSoft). Level 2/3 data products group individual "CCD frames" (Level 1.5) into logical units. There are two different types of Level 2 data products: spectral rasters and slit-jaw image time series. These are the data products that will be most widely used. As calibration procedures improve, these may be regenerated as appropriate.

\subsubsection{Level 2 Spectral Rasters}

Spectral rasters are defined as a series of spectral frames that are

- within one execution of an OBS list

- a contiguous set with identical $\operatorname{sgn}(\delta \mathrm{H})$, in which $\delta \mathrm{H}$ is the value of the perpendicular displacement of the active secondary (controlled by PZT ABC at the FRM and OBS list level)

- if $\delta H=0$ (i.e. sit-and-stare), then all spectra go into one (time series) file

All spectral regions of interest of one raster step are stacked into one "raster" file, including NUV and FUV (i.e. unlike Level 1 these different spectra are now stored into one file). The spectral windows are stacked as extensions (as part of the new FITS 3 standard). Each spectral region within a CRS is stored into a separate extension. Each extension has auxiliary header information that describes time, position, etc.

\subsubsection{Level 2 Slitjaw Images}

All slitjaw (SJI) images of one execution of an OBS list and for one filter-wheel (FW) position and readout region (CRS) are stored as one separate file. These SJI files contain a time series. There are separate SJI files for each filter-wheel choice and for each different CRS region.

\subsubsection{Level 3}

Level 3 data groups the Level 2 data into products that allow easy quick-look and analysis using the IRIS-specific crispex.pro quick-look tool (Vissers and Rouppe van der Voort, 2012). In practice this means that time series of spectral rasters are re-ordered into fourdimensional data cubes (FITS files).

CRISPEX was developed to analyze data taken with the CRISP Fabry-Perot interferometer (Scharmer et al., 2008). This instrument takes two-dimensional images of the Sun within a narrow bandpass centered at a specific wavelength. CRISP is commonly used to perform rapid wavelength scans through a chromospheric line. To facilitate speedy access to the large four-dimensional $(x, y, \lambda, t)$ datasets CRISPEX does not read the data into memory, but reads it from disk as it is accessed. To optimize the speed at which the data is displayed, 
it is stored in two separate formats: $x, y, \lambda, t$ and $\lambda, t, x, y$. This allows for rapid display of both images, $\lambda$-intensity, $\lambda-t$, and $\lambda-x$ plots, allowing easy quick-look access.

To fully exploit CRISPEX, IRIS Level 2 data are rendered into CRISPEX-ready files. The latter are the IRIS Level 3 data products.

In particular, a Level 3 IRIS CRISPEX file will be based on a specific execution of an OBS list and contain either of these:

- one IRIS (synthetic) spectral window stored in $x y \lambda t$ or $\lambda t x y$ format

- all spectral windows stored in $x y \lambda t$ or $\lambda t x y$ format.

Level 3 data is not distributed publicly because the data volume is very large, but can be produced from Level 2 data using SolarSoft (iris_make_fits_level3.pro).

\subsection{Other Products}

Browse products are generated from Level 2 and 3 images. Heliophysics Coverage Registry (HCR) products are derived from the planned operations and the resulting data, and are recorded in the Heliophysics Coverage Registry, which is part of the Heliophysics Event Knowledgebase (Hurlburt et al., 2012).

\subsection{Data Availability}

Data from IRIS are made available to the community through several paths. Level 2 data products are the standard science products. These are available through the IRIS website (iris.Imsal.com) in a manner similar to Hinode, TRACE, and the AIA cutout web services. Level 2 data are also available at the Hinode archive at the University of Oslo. Higher-level descriptions of the data products are maintained within the Heliophysics Coverage Registry and solar events associated with these data products are captured within the Heliophysics Event Registry. IRIS data are available via the Virtual Solar Observatory (Hill et al., 2009) through a variety of avenues.

Level 1 data are available directly from the JSOC using the same procedures as developed for SDO. These Level 1 data are a published series that is either available through the JSOC subscription mechanisms or through the "look-data" web interface.

\section{Numerical Modeling}

\subsection{Overview}

The IRIS investigation includes an extensive radiative-MHD numerical-simulation component to enable full forward-modeling of the domain from the top of the convective envelope to the low corona. Such a comprehensive approach to numerical modeling is critical for a full understanding of the interface region and the diagnostics that cover this region. Advances in algorithms, supercomputing capability, and parallelization techniques now allow such computations at a spatial and temporal resolution that is adequate for comparisons with IRIS observables, including various physical mechanisms that have a critical role in the thermodynamic evolution of the low solar atmosphere.

The rapid advances in computational infrastructure and algorithms have revolutionized the state-of-the-art of numerical simulations and the role these play in interpreting observations. These advances are dramatically reducing the idealizations and simplifications that are 
made to enable the computations. As part of the pre-launch IRIS science investigation, the co-I team has worked towards improving the realism of various numerical models through intensive collaboration and annual workshops.

These efforts have been focused on

- increased spatial resolution,

- wide range of magnetic-field configuration, including small-scale flux emergence,

- increased volume of numerical domain,

- inclusion of non-equilibrium ionization of hydrogen and helium,

- inclusion of effects of ion-neutral interactions in the partially ionized chromosphere,

- inclusion of kinetic effects through a hybrid PIC/MHD approach,

- line formation and interpretation of C II $1335 \AA$ and Mg II h/k 2796/2803 A,

- comparison of physical and diagnostic results from various numerical models.

Despite the advances in numerical modeling, it is clear that simplifying assumptions are still required to enable the calculations. The IRIS modeling philosophy is that comparisons between IRIS (and other) observations and synthetic diagnostics from numerical models are used to determine which physical mechanisms reduce any discrepancies between observations and models, and which assumptions are too idealized and should be relaxed. For example, preliminary studies of the formation of the $\mathrm{Mg}$ II $\mathrm{h}$ and $\mathrm{k}$ lines indicate that they provide a sensitive diagnostic for upper-chromospheric heating and turbulence, and that current models lack the correct amount of heating and turbulence just below the transition region, most likely because time-dependent hydrogen ionization and ambipolar diffusion have not yet been fully taken into account, and/or because the simulations lack sufficient spatial resolution. This combined approach, in which observations and models are continuously compared, guiding the next development steps for the models, is a major part of the IRIS science investigation.

The numerical modeling team of the IRIS science investigation has played a crucial role throughout the development of the IRIS-instrument requirements, for example by assisting with assessing the impact of telescope and instrument design choices in terms of full width half maximum of the Šolc filter, or studying the requirements for spectral resolution in the NUV SG passband. IRIS modeling has helped not only with determining requirements, but also assisted in design choices later in the development program. The IRIS models have also been highly valuable in assisting with pre-launch optimization of observing sequences through the use of the so-called IRIS simulator, which allows a user to observe the models with the same observing tables that IRIS uses. This code is available in the IRIS SolarSoft tree.

\subsection{Publicly Available Models and Articles}

To assist the community with the interpretation of IRIS data, several numerical models computed with the Bifrost code from the University of Oslo are for the first time being publicly released (sdc.uio.no/search/simulations). The numerical models in question have also been used to investigate the line formation of Mg II h and k (Leenaarts et al., 2013a), the relationship between spectral features in the $\mathrm{Mg}$ II h/k lines and physical variables in the chromosphere (Leenaarts et al., 2013b), how IRIS observations of $\mathrm{Mg} \mathrm{II} \mathrm{h/k} \mathrm{can} \mathrm{be} \mathrm{used}$ to diagnose chromospheric conditions (Pereira et al., 2013), the line formation of the C II $1335 \AA$ lines, and the effects of non-equilibrium ionization on the IRIS FUV diagnostics (Olluri, Gudiksen, and Hansteen, 2013). 
Table 11 IRIS Documentation and URLs.

\begin{tabular}{ll}
\hline Description & URL \\
\hline Main website & iris.Imsal.com \\
Operations & iris.Imsal.com/operations.html \\
Data search & iris.Imsal.com/search/ \\
& sdc.uio.no/search/form \\
Recent observations & www.Imsal.com/hek/hcr?cmd=view-recent-events\&instrument=iris \\
IRIS Today & iris.Imsal.com/iristoday \\
Documentation & iris.Imsal.com/documents.html \\
\hline
\end{tabular}

\section{Conclusion}

The IRIS door was opened and the first images and spectra were acquired on 17 July 2013. Since then, IRIS has successfully finished its initial 60-day observing plan, which was focused on obtaining a variety of data sets (sit-and-stare, dense rasters, coarse rasters) for a range of different targets (quiet Sun, active regions, coronal holes, prominences). The IRIS slit-jaw images and spectra reveal a variety of highly dynamic and finely scaled structures, often involving high velocities. Both the FUV and NUV channels of IRIS perform very well, with C II, Si IV, Mg II k, and Mg II h spectra providing a novel window on the intimate coupling between the photosphere, chromosphere, and transition region. Hotter active regions and/or flares also show Fe XII and Fe XXI spectra, extending IRIS's temperature coverage well into the hot corona. The spatial, spectral, and temporal resolution are excellent, reaching $0.33-0.4$ arcsec, $<1 \mathrm{~km} \mathrm{~s}^{-1}$, and down to 1.5 seconds, respectively. The various channels are well co-aligned and the image-stabilization system is excellent. Typical observing sequences take spectra and images at cadences between 3 and 30 seconds. Calibration work to monitor long-term behavior continues, but calibrated data sets are now available to the public without restriction within a few days of observations. Extensive documentation on operations, calibration, data analysis, and numerical simulations is available in the form of so-called IRIS Technical Notes (ITNs) on the iris.Imsal.com website, as outlined in Table 11.

Acknowledgements The effort required to build a mission such as IRIS requires a large, skillful, and dedicated team. We wish to acknowledge many individuals who contributed to the success of IRIS: Geoff Andrews, Nate Caditan, Brock Carpenter, Jay Dusenbury, Cliff Evans, Chuck Fischer, Scott Green, George Dankiewicz, Robert Honeycutt, James Irwin, Harjeet Janda, Dwana Kacensky, Pete Kacensky, Noah Katz, Mike Marticorena, Mark Ridley, John Serafin, David Schiff, Richard Shine, Araya Silpikul, Greg Slater, Shanti Varaitch, Leah Wang, Ross Yamamoto and Kent Zikuhr (Lockheed Martin). We are grateful to Mats Löfdahl for providing his IDL phase-diversity code and teaching us how to use it. We would like to thank Nicholas Chrissotimos, Gregory Frazier, Eric Ianson, Christine Hinkle, and Valerie Mackritis from the NASA GSFC Explorers Office for their support throughout the entire IRIS mission. We would also like to thank Jeff Newmark, Joseph Davila, and Adrian Daw at NASA for their support and encouragement. This work is supported by NASA under contract NNG09FA40C and the Lockheed Martin Independent Research Program. The data downlink to Svalbard is funded by the Norwegian Space Centre (NSC) through an ESA PRODEX contract. We would like to thank Bo Andersen for his efforts in making the NSC downlink support possible.

Open Access This article is distributed under the terms of the Creative Commons Attribution License which permits any use, distribution, and reproduction in any medium, provided the original author(s) and the source are credited.

\section{Appendix: Observing Tables}

The default observing tables are given in Tables 12, 13, and 14 . 
Table 12 IRIS default tables: basic raster modes.

\begin{tabular}{|c|c|c|c|}
\hline OBS number & $\begin{array}{l}\text { Raster step } \\
\text { [arcsec] }\end{array}$ & $\begin{array}{l}\text { Raster size } \\
{\left[\operatorname{arcsec}^{2}\right]}\end{array}$ & Description \\
\hline 1 & 0.33 & $0.3 \times 30$ & Small sit-and-stare \\
\hline 2 & 0.33 & $0.3 \times 60$ & Medium sit-and-stare \\
\hline 3 & 0.33 & $0.3 \times 120$ & Large sit-and-stare \\
\hline 4 & 0.33 & $0.3 \times 175$ & Very large sit-and-stare \\
\hline 5 & 0.33 & $0.33 \times 30$ & Small dense 2-step raster \\
\hline 6 & 0.33 & $0.33 \times 60$ & Medium dense 2-step raster \\
\hline 7 & 0.33 & $0.33 \times 175$ & Very large dense 2 -step raster \\
\hline 8 & 1 & $1 \times 60$ & Medium sparse 2-step raster \\
\hline 9 & 1 & $1 \times 120$ & Large sparse 2-step raster \\
\hline 10 & 1 & $1 \times 175$ & Very large sparse 2 -step raster \\
\hline 11 & 2 & $2 \times 60$ & Medium coarse 2 -step raster \\
\hline 12 & 2 & $2 \times 120$ & Large coarse 2-step raster \\
\hline 13 & 2 & $2 \times 175$ & Very large coarse 2 -step raster \\
\hline 14 & 0.33 & $1 \times 30$ & Small dense 4-step raster \\
\hline 15 & 0.33 & $1 \times 60$ & Medium dense 4-step raster \\
\hline 16 & 0.33 & $1 \times 175$ & Very large dense 4-step raster \\
\hline 17 & 1 & $3 \times 60$ & Medium sparse 4-step raster \\
\hline 18 & 1 & $3 \times 120$ & Large sparse 4-step raster \\
\hline 19 & 1 & $3 \times 175$ & Very large sparse 4-step raster \\
\hline 20 & 2 & $6 \times 60$ & Medium coarse 4-step raster \\
\hline 21 & 2 & $6 \times 120$ & Large coarse 4-step raster \\
\hline 22 & 2 & $6 \times 175$ & Very large coarse 4 -step raster \\
\hline 23 & 0.33 & $2.32 \times 30$ & Small dense 8-step raster \\
\hline 24 & 0.33 & $2.32 \times 60$ & Medium dense 8-step raster \\
\hline 25 & 0.33 & $2.32 \times 175$ & Very large dense 8-step raster \\
\hline 26 & 1 & $7 \times 60$ & Medium sparse 8-step raster \\
\hline 27 & 1 & $7 \times 120$ & Large sparse 8-step raster \\
\hline 28 & 1 & $7 \times 175$ & Very large sparse 8 -step raster \\
\hline 29 & 2 & $14 \times 60$ & Medium coarse 8 -step raster \\
\hline 30 & 2 & $14 \times 120$ & Large coarse 8-step raster \\
\hline 31 & 2 & $14 \times 175$ & Very large coarse 8-step raster \\
\hline 32 & 0.33 & $5 \times 60$ & Medium dense 16-step raster \\
\hline 33 & 0.33 & $5 \times 120$ & Large dense 16-step raster \\
\hline 34 & 0.33 & $5 \times 175$ & Very large dense 16-step raster \\
\hline 35 & 1 & $15 \times 60$ & Medium sparse 16-step raster \\
\hline 36 & 1 & $15 \times 120$ & Large sparse 16 -step raster \\
\hline 37 & 1 & $15 \times 175$ & Very large sparse 16 -step raster \\
\hline 38 & 2 & $30 \times 120$ & Large coarse 16-step raster \\
\hline 39 & 2 & $30 \times 175$ & Very large coarse 16 -step raster \\
\hline 40 & 0.33 & $20.8 \times 120$ & Large dense 64-step raster \\
\hline 41 & 0.33 & $20.8 \times 175$ & Very large dense 64 -step raster \\
\hline 42 & 1 & $63 \times 120$ & Large sparse 64-step raster \\
\hline 43 & 1 & $63 \times 175$ & Very large sparse 64 -step raster \\
\hline 44 & 2 & $126 \times 120$ & Large coarse 64-step raster \\
\hline 45 & 2 & $126 \times 175$ & Very large coarse 64 -step raster \\
\hline 46 & 0.33 & $131.7 \times 175$ & Very large dense raster \\
\hline 47 & 0.33 & $31.35 \times 175$ & Dense synoptic raster \\
\hline 48 & 1 & $35 \times 175$ & Sparse synoptic raster \\
\hline 49 & 2 & $34 \times 175$ & Coarse synoptic raster \\
\hline
\end{tabular}


Table 13 IRIS default tables: SJI, exposure times, rebinning.

\begin{tabular}{|c|c|}
\hline OBS number & Description \\
\hline 0 & C II Si IV Mg II h/k Mg II w \\
\hline 100 & C II Si IV Mg II h/k Mg II w s \\
\hline 200 & C II Si IV Mg II w s \\
\hline 300 & $\mathrm{C}$ II $\mathrm{Mg}$ II $\mathrm{h} / \mathrm{k} \mathrm{Mg}$ II w s \\
\hline 400 & Si IV Mg II h/k Mg II w s \\
\hline 500 & C II Mg II w s \\
\hline 600 & Si IV Mg II w s \\
\hline 700 & $\mathrm{Mg}$ II $\mathrm{h} / \mathrm{k} \mathrm{Mg}$ II w s \\
\hline 800 & Si IV Mg II h/k Mg II w \\
\hline 900 & $\mathrm{C}$ II $\mathrm{Mg}$ II $\mathrm{h} / \mathrm{k} \mathrm{Mg}$ II w \\
\hline 1000 & C II Si IV Mg II w \\
\hline 1100 & C II Si IV Mg II h/k \\
\hline 1200 & C II Si IV \\
\hline 1300 & C II Mg II h/k \\
\hline 1400 & Si IV Mg II h/k \\
\hline 1500 & $\mathrm{C}_{\text {II }}$ \\
\hline 1600 & Si IV \\
\hline 1700 & $\mathrm{Mg}$ II h/k \\
\hline 1800 & Mg II w \\
\hline 1900 & $\mathrm{Mg}$ II $\mathrm{h} / \mathrm{k} \mathrm{Mg}$ II w \\
\hline 0 & Exp time $1 \mathrm{~s}$ \\
\hline 2000 & Exp time $0.5 \mathrm{~s}$ \\
\hline 4000 & Exp time $2 \mathrm{~s}$ \\
\hline 6000 & Exp time $4 \mathrm{~s}$ \\
\hline 8000 & Exp time $8 \mathrm{~s}$ \\
\hline 10000 & Exp time $15 \mathrm{~s}$ \\
\hline 12000 & Exp time $30 \mathrm{~s}$ \\
\hline 0 & Spatial $\times 1$, Spectral $\times 1$ \\
\hline 20000 & Spatial $\times 1$, Spectral $\times 2$ \\
\hline 40000 & Spatial $\times 1$, Spectral $\times 4$ \\
\hline 60000 & Spatial $\times 1$, Spectral $\times 8$ \\
\hline 80000 & Spatial $\times 2$, Spectral $\times 1$ \\
\hline 100000 & Spatial $\times 2$, Spectral $\times 2$ \\
\hline 120000 & Spatial $\times 2$, Spectral $\times 4$ \\
\hline 140000 & Spatial $\times 2$, Spectral $\times 8$ \\
\hline 160000 & Spatial $\times 4$, Spectral $\times 1$ \\
\hline 180000 & Spatial $\times 4$, Spectral $\times 2$ \\
\hline 200000 & Spatial $\times 4$, Spectral $\times 4$ \\
\hline 220000 & Spatial $\times 4$, Spectral $\times 8$ \\
\hline 0 & FUV spectrally rebinned $\times 2$ \\
\hline 250000 & FUV spectrally rebinned $\times 2$ \\
\hline 500000 & FUV spectrally rebinned $\times 4$ \\
\hline 750000 & FUV spectrally rebinned $\times 8$ \\
\hline
\end{tabular}


Table 14 IRIS default tables: SJI cadence, compression, line lists.

\begin{aligned} & \hline OBS number Description \\ & \hline 0 SJI cadence $10 \mathrm{~s}(60$ s for slow $) \\ & 1000000$ SJI cadence $0.25 \times$ faster \\ & 2000000 SJI cadence $0.5 \times$ faster \\ & 3000000 SJI cadence $3 \times$ faster \\ & 4000000 SJI cadence $10 \times$ faster \\ & 0 Default lossy compression \\ & 10000000 Lossless compression \\ & 0 Large line list \\ & 20000000 Medium line list \\ & 40000000 Small line list \\ & 60000000 Flare line list \\ & 80000000 Full readout \\ & \hline\end{aligned}

\section{References}

Allen, C.W.: 1964, Astrophysical Quantities, Athelone, London. ADS.

Allen, M.S., McAllister, H.C.: 1978, Observations of the Mg I and II resonance lines in an active region. Solar Phys. 60, 251. DOI. ADS.

Arber, T.D., Haynes, M., Leake, J.E.: 2007, Emergence of a flux tube through a partially ionized solar atmosphere. Astrophys. J. 666, 541. DOI. ADS.

Axford, W.I., McKenzie, J.F., Sukhorukova, G.V., Banaszkiewicz, M., Czechowski, A., Ratkiewicz, R.: 1999, Acceleration of the high speed solar wind in coronal holes. Space Sci. Rev. 87, 25. DOI. ADS.

Bates, B., Bradley, D.J., McKeith, C.D., McKeith, N.E.: 1969, Fabry-Perot interferograms of the solar Mg II doublet and XUV solar images obtained during a stabilized skylark rocket flight. Nature 224, 161. DOI. ADS.

Beck, C., Schmidt, W., Rezaei, R., Rammacher, W.: 2008, The signature of chromospheric heating in Ca II H spectra. Astron. Astrophys. 479, 213. DOI. ADS.

Berger, T., Mudge, J., Holmes, B., Searcy, P., Wuelser, J.P., Lemen, J., Title, A.: 2012, Design and fabrication of the near-ultraviolet birefringent Solc filter for the NASA IRIS solar physics mission. Proc. SPIE 8486. DOI. ADS.

Billings, D.E., Roussel-Dupre, R., Francis, M.H.: 1977, Dynamical implications of SI IV line profiles from OSO-8 observations. Solar Phys. 55, 287. DOI. ADS.

Bonnet, R.M., Lemaire, P., Vial, J.C., Artzner, G., Gouttebroze, P., Jouchoux, A., Vidal-Madjar, A., Leibacher, J.W., Skumanich, A.: 1978, The LPSP instrument on OSO 8. II - In-flight performance and preliminary results. Astrophys. J. 221, 1032. DOI. ADS.

Bryans, P., Young, P.R., Doschek, G.A.: 2010, Multiple component outflows in an active region observed with the EUV Imaging Spectrometer on Hinode. Astrophys. J. 715, 1012. DOI. ADS.

Cally, P.S., Goossens, M.: 2008, Three-dimensional MHD wave propagation and conversion to Alfvén waves near the solar surface. I. Direct numerical solution. Solar Phys. 251, 251. DOI. ADS.

Cavallini, F.: 2006, IBIS: A new post-focus instrument for solar imaging spectroscopy. Solar Phys. 236, 415. DOI. ADS.

Chae, J.: 2004, Flat-fielding of solar $\mathrm{H} \alpha$ observations using relatively shifted images. Solar Phys. 221, 1. DOI. ADS.

Cirtain, J.W., Golub, L., Winebarger, A.R., de Pontieu, B., Kobayashi, K., Moore, R.L., Walsh, R.W., Korreck, K.E., Weber, M., McCauley, P., Title, A., Kuzin, S., Deforest, C.E.: 2013, Energy release in the solar corona from spatially resolved magnetic braids. Nature 493, 501. DOI. ADS.

Cranmer, S.R., van Ballegooijen, A.A., Edgar, R.J.: 2007, Self-consistent coronal heating and solar wind acceleration from anisotropic magnetohydrodynamic turbulence. Astrophys. J. 171, 520. DOI. ADS.

Culhane, J.L., Harra, L.K., James, A.M., Al-Janabi, K., Bradley, L.J., Chaudry, R.A., Rees, K., Tandy, J.A., Thomas, P., Whillock, M.C.R., Winter, B., Doschek, G.A., Korendyke, C.M., Brown, C.M., Myers, S., Mariska, J., Seely, J., Lang, J., Kent, B.J., Shaughnessy, B.M., Young, P.R., Simnett, G.M., Castelli, 
C.M., Mahmoud, S., Mapson-Menard, H., Probyn, B.J., Thomas, R.J., Davila, J., Dere, K., Windt, D., Shea, J., Hagood, R., Moye, R., Hara, H., Watanabe, T., Matsuzaki, K., Kosugi, T., Hansteen, V., Wikstol, Ø.: 2007, The EUV imaging spectrometer for Hinode. Solar Phys. 243, 19. DOI. ADS.

De Pontieu, B., McIntosh, S.W., Carlsson, M., Hansteen, V.H., Tarbell, T.D., Schrijver, C.J., Title, A.M., Shine, R.A., Tsuneta, S., Katsukawa, Y., Ichimoto, K., Suematsu, Y., Shimizu, T., Nagata, S.: 2007a, Chromospheric Alfvénic waves strong enough to power the solar wind. Science 318, 1574. DOI. ADS.

De Pontieu, B., Hansteen, V.H., Rouppe van der Voort, L., van Noort, M., Carlsson, M.: 2007b, Highresolution observations and modeling of dynamic fibrils. Astrophys. J. 655, 624. DOI. ADS.

De Pontieu, B., McIntosh, S.W., Hansteen, V.H., Schrijver, C.J.: 2009, Observing the roots of solar coronal heating in the chromosphere. Astrophys. J. Lett. 701, L1. DOI. ADS.

De Pontieu, B., McIntosh, S.W., Carlsson, M., Hansteen, V.H., Tarbell, T.D., Boerner, P., Martinez-Sykora, J., Schrijver, C.J., Title, A.M.: 2011, The origins of hot plasma in the solar corona. Science 331, 55. DOI. ADS.

De Pontieu, B., Carlsson, M., Rouppe van der Voort, L.H.M., Rutten, R.J., Hansteen, V.H., Watanabe, H.: 2012, Ubiquitous torsional motions in type II spicules. Astrophys. J. Lett. 752, L12. DOI. ADS.

Dere, K.P., Bartoe, J.-D.F., Brueckner, G.E.: 1984, High-resolution telescope and spectrograph observations of the quiet solar chromosphere and transition zone. Astrophys. J. 281, 870. DOI. ADS.

Doschek, G.A., Feldman, U.: 1977, High-resolution spectra of the solar Mg II H and K lines from SKYLAB. Astrophys. J. Suppl. 35, 471. DOI. ADS.

Fontenla, J.M., Peterson, W.K., Harder, J.: 2008, Chromospheric heating by the Farley-Buneman instability. Astron. Astrophys. 480, 839. DOI. ADS.

Fredga, K.: 1969, Spectroheliograms in the Mg II line at 2795.5 A. Solar Phys. 9, 358. DOI. ADS.

Gogoberidze, G., Voitenko, Y., Poedts, S., Goossens, M.: 2009, Farley-Buneman instability in the solar chromosphere. Astrophys. J. Lett. 706, L12. DOI. ADS.

Goodman, M.L., Kazeminezhad, F.: 2010, Simulation of magnetohydrodynamic shock wave generation, propagation, and heating in the photosphere and chromosphere using a complete electrical conductivity tensor. Astrophys. J. 708, 268. DOI. ADS.

Guerreiro, N., Hansteen, V., De Pontieu, B.: 2013, The cycling of material between the solar corona and chromosphere. Astrophys. J. 769, 47. DOI. ADS.

Handy, B.N., Acton, L.W., Kankelborg, C.C., Wolfson, C.J., Akin, D.J., Bruner, M.E., Caravalho, R., Catura, R.C., Chevalier, R., Duncan, D.W., Edwards, C.G., Feinstein, C.N., Freeland, S.L., Friedlaender, F.M., Hoffmann, C.H., Hurlburt, N.E., Jurcevich, B.K., Katz, N.L., Kelly, G.A., Lemen, J.R., Levay, M., Lindgren, R.W., Mathur, D.P., Meyer, S.B., Morrison, S.J., Morrison, M.D., Nightingale, R.W., Pope, T.P., Rehse, R.A., Schrijver, C.J., Shine, R.A., Shing, L., Strong, K.T., Tarbell, T.D., Title, A.M., Torgerson, D.D., Golub, L., Bookbinder, J.A., Caldwell, D., Cheimets, P.N., Davis, W.N., Deluca, E.E., McMullen, R.A., Warren, H.P., Amato, D., Fisher, R., Maldonado, H., Parkinson, C.: 1999, The transition region and coronal explorer. Solar Phys. 187, 229. DOI. ADS.

Hansteen, V.H., De Pontieu, B., Rouppe van der Voort, L., van Noort, M., Carlsson, M.: 2006, Dynamic fibrils are driven by magnetoacoustic shocks. Astrophys. J. Lett. 647, L73. DOI. ADS.

Harra, L.K., Hara, H., Imada, S., Young, P.R., Williams, D.R., Sterling, A.C., Korendyke, C., Attrill, G.D.R.: 2007, Coronal dimming observed with Hinode: Outflows related to a coronal mass ejection. Publ. Astron. Soc. Japan 59, 801. ADS.

Hertz, E.N., Cheimets, P.N., Podgorski, W.A., Perry, T., Park, S.C., Bergner, H.W., Gates, R., Marquez, V., Honsa, M.F.: 2012, Design, analysis, and performance verification of the interface region imaging spectrograph (IRIS) telescope primary mirror assembly. Proc. SPIE 8443. DOI. ADS.

Hill, F., Martens, P., Yoshimura, K., Gurman, J., Hourclé, J., Dimitoglou, G., Suárez-Solá, I., Wampler, S., Reardon, K., Davey, A., Bogart, R.S., Tian, K.Q.: 2009, The virtual solar observatory: A resource for international heliophysics research. Earth Moon Planets 104, 315. DOI. ADS.

Hurlburt, N., Cheung, M., Schrijver, C., Chang, L., Freeland, S., Green, S., Heck, C., Jaffey, A., Kobashi, A., Schiff, D., Serafin, J., Seguin, R., Slater, G., Somani, A., Timmons, R.: 2012, Heliophysics event knowledgebase for the Solar Dynamics Observatory (SDO) and beyond. Solar Phys. 275, 67. DOI. ADS.

Jefferies, S.M., McIntosh, S.W., Armstrong, J.D., Bogdan, T.J., Cacciani, A., Fleck, B.: 2006, Magnetoacoustic portals and the basal heating of the solar chromosphere. Astrophys. J. Lett. 648, L151. DOI. ADS.

Kato, Y., Steiner, O., Steffen, M., Suematsu, Y.: 2011, Excitation of slow modes in network magnetic elements through magnetic pumping. Astrophys. J. Lett. 730, L24. DOI. ADS.

Kingston, A.E., Doyle, J.G., Dufton, P.L., Gurman, J.B.: 1982, An emission measure analysis of two sunspots observed by the UVSP instrument on the SMM spacecraft. Solar Phys. 81, 47. DOI. ADS.

Kohl, J.L., Parkinson, W.H.: 1976, The Mg II H and K lines. I - Absolute center and limb measurements of the solar profiles. Astrophys. J. 205, 599. DOI. ADS. 
Kosugi, T., Matsuzaki, K., Sakao, T., Shimizu, T., Sone, Y., Tachikawa, S., Hashimoto, T., Minesugi, K., Ohnishi, A., Yamada, T., Tsuneta, S., Hara, H., Ichimoto, K., Suematsu, Y., Shimojo, M., Watanabe, T., Shimada, S., Davis, J.M., Hill, L.D., Owens, J.K., Title, A.M., Culhane, J.L., Harra, L.K., Doschek, G.A., Golub, L.: 2007, The Hinode (Solar-B) mission: An overview. Solar Phys. 243, 3. DOI. ADS.

Leenaarts, J., Pereira, T.M.D., Carlsson, M., Uitenbroek, H., De Pontieu, B.: 2013a, The formation of IRIS diagnostics. I. A quintessential model atom of $\mathrm{Mg}$ II and general formation properties of the $\mathrm{Mg}$ II $\mathrm{h}$ and $\mathrm{k}$ lines. Astrophys. J. 772, 89. DOI. ADS.

Leenaarts, J., Pereira, T.M.D., Carlsson, M., Uitenbroek, H., De Pontieu, B.: 2013b, The formation of IRIS diagnostics. II. The formation of the Mg II h and k lines in the solar atmosphere. Astrophys. J. 772, 90. DOI. ADS.

Lemaire, P.: 1969, High resolution balloon spectra of the Sun in the Mg II doublet lines II. Astrophys. J. Lett. 3, L43. ADS.

Lemaire, P., Skumanich, A.: 1973, Magnesium II doublet profiles of chromospheric inhomogeneities at the center of the solar disk. Astron. Astrophys. 22, 61. ADS.

Lemen, J.R., Title, A.M., Akin, D.J., Boerner, P.F., Chou, C., Drake, J.F., Duncan, D.W., Edwards, C.G., Friedlaender, F.M., Heyman, G.F., Hurlburt, N.E., Katz, N.L., Kushner, G.D., Levay, M., Lindgren, R.W., Mathur, D.P., McFeaters, E.L., Mitchell, S., Rehse, R.A., Schrijver, C.J., Springer, L.A., Stern, R.A., Tarbell, T.D., Wuelser, J.-P., Wolfson, C.J., Yanari, C., Bookbinder, J.A., Cheimets, P.N., Caldwell, D., Deluca, E.E., Gates, R., Golub, L., Park, S., Podgorski, W.A., Bush, R.I., Scherrer, P.H., Gummin, M.A., Smith, P., Auker, G., Jerram, P., Pool, P., Soufli, R., Windt, D.L., Beardsley, S., Clapp, M., Lang, J., Waltham, N.: 2012, The Atmospheric Imaging Assembly (AIA) on the Solar Dynamics Observatory (SDO). Solar Phys. 275, 17. DOI. ADS.

Lites, B.W., Kubo, M., Socas-Navarro, H., Berger, T., Frank, Z., Shine, R., Tarbell, T., Title, A., Ichimoto, K., Katsukawa, Y., Tsuneta, S., Suematsu, Y., Shimizu, T., Nagata, S.: 2008, The horizontal magnetic flux of the quiet-Sun internetwork as observed with the Hinode spectro-polarimeter. Astrophys. J. 672, 1237. DOI. ADS.

Liu, W., Title, A.M., Zhao, J., Ofman, L., Schrijver, C.J., Aschwanden, M.J., De Pontieu, B., Tarbell, T.D.: 2011 , Direct imaging of quasi-periodic fast propagating waves of $\approx 2000 \mathrm{~km} \mathrm{~s}^{-1}$ in the low solar corona by the solar dynamics observatory atmospheric imaging assembly. Astrophys. J. Lett. 736, L13. DOI. ADS.

Löfdahl, M.G., Scharmer, G.B.: 1994, Wavefront sensing and image restoration from focused and defocused solar images. Astron. Astrophys. Suppl. 107, 243. ADS.

Martínez-Sykora, J., De Pontieu, B., Hansteen, V.: 2012, Two-dimensional radiative magnetohydrodynamic simulations of the importance of partial ionization in the chromosphere. Astrophys. J. 753, 161. DOI. ADS.

McIntosh, S.W., De Pontieu, B.: 2012, Estimating the "dark" energy content of the solar corona. Astrophys. J. 761, 138. DOI. ADS.

McIntosh, S.W., de Pontieu, B., Carlsson, M., Hansteen, V., Boerner, P., Goossens, M.: 2011, Alfvénic waves with sufficient energy to power the quiet solar corona and fast solar wind. Nature 475, 477. DOI. ADS.

McIntosh, S.W., Tian, H., Sechler, M., De Pontieu, B.: 2012, On the Doppler velocity of emission line profiles formed in the "coronal contraflow" that is the chromosphere-corona mass cycle. Astrophys. J. 749, 60. DOI. ADS.

Morrill, J.S., Korendyke, C.M.: 2008, High-resolution center-to-limb variation of the quiet solar spectrum near Mg II. Astrophys. J. 687, 646. DOI. ADS.

Okamoto, T.J., De Pontieu, B.: 2011, Propagating waves along spicules. Astrophys. J. Lett. 736, L24. DOI. ADS.

Olluri, K., Gudiksen, B.V., Hansteen, V.H.: 2013, Non-equilibrium ionization effects on the density line ratio diagnostics of O IV. Astrophys. J. 767, 43. DOI. ADS.

Park, S.C., Yanari, C.H., Cheimets, P.N., Podgorski, W.A., Wuelser, J.-P.: 2012, Thermal design of interface region imaging spectrograph (IRIS) ULE primary mirror. Proc. SPIE 8443. DOI. ADS.

Pereira, T.M.D., Leenaarts, J., De Pontieu, B., Carlsson, M., Uitenbroek, H.: 2013, The formation of IRIS diagnostics. III. Near-ultraviolet spectra and images. Astrophys. J. 778, 143. DOI. ADS.

Pesnell, W.D., Thompson, B.J., Chamberlin, P.C.: 2012, The Solar Dynamics Observatory (SDO). Solar Phys. 275, 3. DOI. ADS.

Podgorski, W.A., Cheimets, P.N., Golub, L., Lemen, J.R., Title, A.M.: 2012, Design, performance prediction, and measurements of the interface region imaging spectrograph (IRIS) telescope. Proc. SPIE 8443. DOI. ADS.

Poland, A.I., Tandberg-Hanssen, E.: 1983, Physical conditions in a quiescent prominence derived from UV spectra obtained with the UVSP instrument on the SMM. Solar Phys. 84, 63. DOI. ADS.

Rice, R.F., Plaunt, J.R.: 1971, Adaptive variable-length coding for efficient compression of spacecraft television data. IEEE Trans. Commun. Technol. 19, 889. DOI. 
Rouppe van der Voort, L.H.M., De Pontieu, B., Hansteen, V.H., Carlsson, M., van Noort, M.: 2007, Magnetoacoustic shocks as a driver of quiet-Sun mottles. Astrophys. J. Lett. 660, L169. DOI. ADS.

Roussel-Dupre, D., Shine, R.A.: 1982, Evidence of redshifts in the average solar line profiles of C IV and SI IV from OSO-8 observations. Solar Phys. 77, 329. DOI. ADS.

Samain, D., Lemaire, P.: 1985, Balloon-borne ultraviolet solar telescope and high resolution Echellespectrograph - Instrumentation and first results. Astrophys. Space Sci. 115, 227. DOI. ADS.

Scharmer, G.B., Bjelksjo, K., Korhonen, T.K., Lindberg, B., Petterson, B.: 2003, The 1-meter Swedish solar telescope. In: Keil, S.L., Avakyan, S.V. (eds.) Proc. SPIE 4853, 341. ADS.

Scharmer, G.B., Narayan, G., Hillberg, T., de la Cruz Rodríguez, J., Löfdahl, M.G., Kiselman, D., Sütterlin, P., van Noort, M., Lagg, A.: 2008, CRISP spectropolarimetric imaging of penumbral fine structure. Astrophys. J. Lett. 689, L69. DOI. ADS.

Scherrer, P.H., Schou, J., Bush, R.I., Kosovichev, A.G., Bogart, R.S., Hoeksema, J.T., Liu, Y., Duvall, T.L., Zhao, J., Title, A.M., Schrijver, C.J., Tarbell, T.D., Tomczyk, S.: 2012, The Helioseismic and Magnetic Imager (HMI) investigation for the Solar Dynamics Observatory (SDO). Solar Phys. 275, 207. DOI. ADS.

Sekse, D.H., Rouppe van der Voort, L., De Pontieu, B., Scullion, E.: 2013, Interplay of three kinds of motion in the disk counterpart of type II spicules: Upflow, transversal, and torsional motions. Astrophys. J. 769, 44. DOI. ADS.

Staath, E., Lemaire, P.: 1995, High resolution profiles of the Mg II H and Mg II K lines. Astron. Astrophys. 295, 517. ADS.

Straus, T., Fleck, B., Jefferies, S.M., Cauzzi, G., McIntosh, S.W., Reardon, K., Severino, G., Steffen, M.: 2008, The energy flux of internal gravity waves in the lower solar atmosphere. Astrophys. J. Lett. 681, L125. DOI. ADS.

Su, J.T., Liu, Y., Liu, S., Zhang, Y.Z., Zhao, H., Xu, H.Q., Xie, W.B.: 2013, Simultaneous observation of solar oscillations associated with coronal loops from the photosphere to the corona. Astrophys. J. 762, 42. DOI. ADS.

Testa, P., De Pontieu, B., Martínez-Sykora, J., DeLuca, E., Hansteen, V., Cirtain, J., Winebarger, A., Golub, L., Kobayashi, K., Korreck, K., Kuzin, S., Walsh, R., DeForest, C., Title, A., Weber, M.: 2013, Observing coronal nanoflares in active region moss. Astrophys. J. Lett. 770, L1. DOI. ADS.

Tian, H., McIntosh, S.W., De Pontieu, B., Martínez-Sykora, J., Sechler, M., Wang, X.: 2011, Two components of the solar coronal emission revealed by extreme-ultraviolet spectroscopic observations. Astrophys. J. 738, 18. DOI. ADS.

Tian, H., McIntosh, S.W., Xia, L., He, J., Wang, X.: 2012, What can we learn about solar coronal mass ejections, coronal dimmings, and extreme-ultraviolet jets through spectroscopic observations? Astrophys. J. 748, 106. DOI. ADS.

Tomczyk, S., McIntosh, S.W.: 2009, Time-distance seismology of the solar corona with CoMP. Astrophys. J. 697, 1384. DOI. ADS.

Tomczyk, S., McIntosh, S.W., Keil, S.L., Judge, P.G., Schad, T., Seeley, D.H., Edmondson, J.: 2007, Alfvén waves in the solar corona. Science 317, 1192. DOI. ADS.

Tu, C.-Y., Marsch, E.: 1997, Two-fluid model for heating of the solar corona and acceleration of the solar wind by high-frequency Alfvén waves. Solar Phys. 171, 363. ADS.

Verwichte, E., Van Doorsselaere, T., White, R.S., Antolin, P.: 2013, Statistical seismology of transverse waves in the solar corona. Astron. Astrophys. 552, A138. DOI. ADS.

Vissers, G., Rouppe van der Voort, L.: 2012, Flocculent flows in the chromospheric canopy of a sunspot. Astrophys. J. 750, 22. DOI. ADS.

Wedemeyer-Böhm, S., Scullion, E., Steiner, O., Rouppe van der Voort, L., de La Cruz Rodriguez, J., Fedun, V., Erdélyi, R.: 2012, Magnetic tornadoes as energy channels into the solar corona. Nature 486, 505. DOI. ADS.

West, E., Cirtain, J., Kobayashi, K., Davis, J., Gary, A., Adams, M.: 2011, Mg II observations using the MSFC solar ultraviolet magnetograph. In: Proc. SPIE 8148. DOI. ADS.

Wilhelm, K., Curdt, W., Marsch, E., Schühle, U., Lemaire, P., Gabriel, A., Vial, J.-C., Grewing, M., Huber, M.C.E., Jordan, S.D., Poland, A.I., Thomas, R.J., Kühne, M., Timothy, J.G., Hassler, D.M., Siegmund, O.H.W.: 1995, SUMER - solar ultraviolet measurements of emitted radiation. Solar Phys. 162, 189. DOI. ADS.

Winebarger, A.R., Walsh, R.W., Moore, R., De Pontieu, B., Hansteen, V., Cirtain, J., Golub, L., Kobayashi, K., Korreck, K., DeForest, C., Weber, M., Title, A., Kuzin, S.: 2013, Detecting nanoflare heating events in subarcsecond inter-moss loops using Hi-C. Astrophys. J. 771, 21. DOI. ADS.

Woodgate, B.E., Brandt, J.C., Kalet, M.W., Kenny, P.J., Tandberg-Hanssen, E.A., Bruner, E.C., Beckers, J.M., Henze, W., Knox, E.D., Hyder, C.L.: 1980, The ultraviolet spectrometer and polarimeter on the solar maximum mission. Solar Phys. 65, 73. DOI. ADS. 
Wülser, J.-P., Title, A.M., Lemen, J.R., De Pontieu, B., Kankelborg, C.C., Tarbell, T.D., Berger, T.E., Golub, L., Kushner, G.D., Chou, C.Y., Weingrod, I., Holmes, B., Mudge, J., Podgorski, W.A.: 2012, The interface region imaging spectrograph for the IRIS Small Explorer mission. In: Proc. SPIE 8443. DOI. ADS.

Young, P.R., Doschek, G.A., Warren, H.P., Hara, H.: 2013, Properties of a solar flare kernel observed by Hinode and SDO. Astrophys. J. 766, 127. DOI. ADS. 